\title{
Existence and multiplicity of positive solutions for fractional Laplacian systems with nonlinear coupling
}

\author{
Guofeng Che and Haibo Chen \\ School of Mathematics and Statistics, \\ Central South University, Changsha, Hunan, 410083, P. R. China \\ Tsung-fang $\mathrm{Wu}^{*}$ \\ Department of Applied Mathematics \\ National University of Kaohsiung, Kaohsiung, Taiwan
}

\begin{abstract}
It is well known that a single nonlinear fractional Schrödinger equation with a potential $V(x)$ may have a positive solution that is concentrated at the nondegenerate minimum point of $V(x)$ as the positive parameter $\varepsilon$ sufficiently small (see [11, 14, 22]). While in this paper, we can find two different positive solutions for weakly coupled fractional Schrödinger systems with two potentials $V_{1}(x)$ and $V_{2}(x)$ having the same minimum point and these positive solutions are concentrated at this minimum point. In fact that by using the energy estimates, Nehari manifold technique and the Lusternik-Schnirelmann theory of critical points, we obtain the multiplicity results for a class of weakly coupled fractional Schrödinger system. Furthermore, the existence and nonexistence of least energy positive solutions are also explored.
\end{abstract}

Keywords: Fractional Laplacian systems; Lusternik-Schnirelmann theory; Positive solutions; Variational methods

2010 Mathematics Subject Classification. Primary: 35J50, 35A01; Secondary: $35 \mathrm{~B} 38$

\section{Introduction}

In this paper, we are concerned with the following nonlinear systems of two weakly coupled fractional Schrödinger equations with nonconstant potentials:

$$
\begin{cases}\varepsilon^{2 \alpha}(-\Delta)^{\alpha} u+V_{1}(x) u=\left(\mu_{1}|u|^{2 p}+\beta(x)|u|^{q-1}|v|^{q+1}\right) u, & \text { in } \mathbb{R}^{N} \\ \varepsilon^{2 \alpha}(-\Delta)^{\alpha} v+V_{2}(x) v=\left(\mu_{2}|v|^{2 p}+\beta(x)|v|^{q-1}|u|^{q+1}\right) v, & \text { in } \mathbb{R}^{N} \\ 0 \leq u, v \in H^{\alpha}\left(\mathbb{R}^{N}\right), & \text { in } \mathbb{R}^{N}\end{cases}
$$

where $\alpha \in(0,1), 0<q \leq p \leq 1$ if $N \leq 4 \alpha$ and $0<q \leq p<\frac{2 \alpha}{N-2 \alpha}$ if $N>4 \alpha, 0<\varepsilon \ll 1$ is a small parameter and $\mu_{l}, l=1,2$, are positive constants. Throughout this paper, we assume that the potentials $V_{1}(x), V_{2}(x)$ and the coupling function $\beta(x)$ satisfy the following hypotheses:

$\left(D_{1}\right) V_{1}(x)$ and $V_{2}(x)$ are bounded and uniformly continuous functions on $\mathbb{R}^{N}$;

G. Che was supported by the Fundamental Research Funds for the Central Universities of Central South University (Grant No. 2017zzts058). H. Chen was supported by the National Natural Science Foundation of China (Grant No. 11671403). T.-F. Wu was supported in part by the Ministry of Science and Technology, Taiwan (Grant No. 106-2115M-390-001-MY2) and the National Center for Theoretical Sciences, Taiwan.

* Corresponding author. E-mail addresses: tfwu@nuk.edu.tw (T.-F. Wu).

E-mail addresses: cheguofeng222@163.com (G. Che), math_chb@163.com (H. Chen). 
$\left(D_{2}\right)$ the potentials $V_{1}(x)$ and $V_{2}(x)$ have isolated gobal minimum points at $z_{1,1}, z_{1,2}, \ldots, z_{1, k}$ and $z_{2,1}, z_{2,2}, \ldots, z_{2, \ell}$, respectively. Here, an isolated gobal minimum point $z_{1, i}$ of $V_{1}(x)$ (similarly to $z_{2, j}$ of $V_{2}(x)$ ) means that $z_{1, i}$ is the unique minimum point of $V_{1}(x)$ in $B_{r_{0}}\left(z_{1, i}\right)$ (a neighborhood of $\left.z_{1, i}\right)$, where $r_{0}$ is a positive constant. Moreover,

$$
V_{1}\left(z_{1, i}\right)=\lambda_{1} \equiv \min \left\{V_{1}(x): x \in \mathbb{R}^{N}\right\}>0 \text { for all } i=1,2 \ldots, k
$$

and

$$
V_{2}\left(z_{2, j}\right)=\lambda_{2} \equiv \min \left\{V_{2}(x): x \in \mathbb{R}^{N}\right\}>0 \text { for all } j=1,2 \ldots, \ell \text {; }
$$

$\left(D_{3}\right) \beta(x)$ is a nonpositive, bounded and uniformly continuous function on $\mathbb{R}^{N}$;

$\left(D_{4}\right)$ there exist a positive integer $1 \leq m \leq \min \{k, \ell\}$ and a constant $c_{0}>0$ such that $z_{1, i}=z_{2, j}$ and $\beta(x) \leq-c_{0}$ for all $x \in B_{r_{0}}\left(z_{1, i}\right)$ for all $1 \leq i, j \leq m$, where $r_{0}>0$ as in condition $\left(D_{2}\right)$.

In recent years great interest has been devoted to the study of elliptic equations involving the fractional Laplacian operator $(-\triangle)^{\alpha}, \alpha \in(0,1)$. This type of operators appears in a quite natural way in many different applications, such as, fractional quantum mechanics, geophysical fluid dynamics, population dynamics, phase transitions, flames propagation, anomalous diffusion, crystal dislocation, materials science, water waves and soft thin films. For more details about the applications, we refer the readers to [18, 29, 30] and the references therein.

Much attention has been paid to the single fractional Laplacian equation:

$$
\varepsilon^{2 \alpha}(-\triangle)^{\alpha} u+V(x) u=f(x, u), \quad \text { in } \mathbb{R}^{N} .
$$

Eq. (1.1) is related to the stationary analogue of the fractional Schrödinger equation:

$$
i \varepsilon \frac{\partial u}{\partial t}=\varepsilon^{2 \alpha}(-\triangle)^{\alpha} u+V(x) u-f(x, u) \quad \text { in } \mathbb{R}^{N},
$$

which was introduced by [29, 30] through expanding the Feynman path integral from the Brownian-like to the Lévy-like quantum mechanical paths. In the past several decades, with the aid of variational methods, the existence, nonexistence, multiplicity, uniqueness, regularity and the asymptotic decay properties of solutions for Eq. (1.1) have been obtained under various hypotheses on the potential $V(x)$ and the nonlinearity $f(x, u)$, see $[11,14,17,20,22,40]$ and the references therein. For instance, Frank, Lenzmann and Silvestre 20] studied the following single fractional Schrödinger equation:

$$
\begin{cases}(-\triangle)^{\alpha} u+\lambda_{l} u=\mu_{l}|u|^{2 p} u, & \text { in } \mathbb{R}^{N}, \\ u \in H^{\alpha}\left(\mathbb{R}^{N}\right), \quad u>0, & \text { in } \mathbb{R}^{N},\end{cases}
$$

where $l=1,2$, and obtained the following properties of the ground states for Eq. $\left(\widehat{E}_{l}\right)$.

Proposition 1.1 Let $N \geq 1, \alpha \in(0,1)$ and $0<p<\frac{2_{\alpha}^{*}-2}{2}$, where

$$
2_{\alpha}^{*}= \begin{cases}\frac{2 N}{N-2 \alpha}, & \text { if } N>2 \alpha \\ +\infty, & \text { if } N \leq 2 \alpha\end{cases}
$$

Then the following hold:

(i) (Uniqueness) The ground state solution $U \in H^{\alpha}\left(\mathbb{R}^{N}\right)$ for Eq. $\left(\widehat{E}_{l}\right)$ is unique.

(ii) (Symmetry, regularity and decay) $U(x)$ is radial, positive and strictly decreasing in $|x|$. Moreover, the function $U(x)$ belongs to $H^{2 \alpha+1}\left(\mathbb{R}^{N}\right) \cap C^{\infty}\left(\mathbb{R}^{N}\right)$ and satisfies

$$
\frac{C_{1}}{1+|x|^{N+2 \alpha}} \leq U(x) \leq \frac{C_{2}}{1+|x|^{N+2 \alpha}}, \forall x \in \mathbb{R}^{N}
$$


with some constants $C_{2} \geq C_{1}>0$.

(iii) (Non-degeneracy) The linearized operator $L_{0}=(-\triangle)^{\alpha}+1-(2 p+1) U^{2 p}$ is non-degenerate, i.e., its kernel is given by

$$
\operatorname{ker} L_{0}=\operatorname{span}\left\{\partial_{x_{1}} U, \cdots, \partial_{x_{N}} U\right\} .
$$

Very recently, many researchers have paid attention to the following Schrödinger system with constant potentials and $\beta$ is a real constant, i.e.

$$
\begin{cases}-\varepsilon^{2} \Delta u+u=\left(|u|^{2 p}+\beta|u|^{p-1}|v|^{p+1}\right) u, & \text { in } \mathbb{R}^{N} \\ -\varepsilon^{2} \Delta v+\omega^{2} v=\left(|v|^{2 p}+\beta|v|^{p-1}|u|^{p+1}\right) v, & \text { in } \mathbb{R}^{N}\end{cases}
$$

which arises as a model for propagation of polarized laser beams in briefringent Kerr medium in nonlinear optics (see [4, 19, 28]). With the help of the critical point theory and the variational methods, there have been lots of results about the existence and multiplicity of nontrivial solutions for system (1.2), see [10, 21, 38] and the references therein. In the case of $p=1, N=2,3, \beta \in \mathbb{R}$ and the potentials may be not constants, we may get the following system:

$$
\begin{cases}-\varepsilon^{2} \Delta u+V_{1}(x) u=\mu_{1} u^{3}+\beta u v^{2}, & \text { in } \mathbb{R}^{N} \\ -\varepsilon^{2} \Delta v+V_{2}(x) v=\mu_{2} v^{3}+\beta v u^{2}, & \text { in } \mathbb{R}^{N}\end{cases}
$$

which appears in the Hartree-Fock theory for a double condensate, i.e., a binary mixture of BoseEinstein condensates in two different hyperfine states $|1\rangle$ and $|2\rangle$ (cf. [16, 25, 42]). Physically, $u$ and $v$ are the corresponding condensate amplitudes, $\varepsilon^{2}=\frac{h^{2}}{2 m}$ and $\mu_{j}=-\left(N_{j}-1\right) U_{j j}$, where $h$ is Planck constant, $m$ is the atom mass, $N_{j}$ is a fixed number of atoms in the hyperfine state $|j\rangle$. Recently, various results on the existence and concentration of solutions for system (1.3) have been obtained, we refer the readers to [26, 31, 32, 33, 39] and the references therein. Peng and Li [39] obtained the existence of multi-spike vector solutions for system system (1.3) with $\beta \neq 0$. Moreover, the attractive phenomenon for $\beta<0$ and the repulsive phenomenon for $\beta>0$ are also explored by the authors. Lin and $\mathrm{Wu}$ [33], use energy estimates and category theory to prove the nonuniqueness theorem, provided $\beta<0$. Lin and Wei [31] proved that there exists $\beta_{0} \in\left(0, \sqrt{\mu_{1} \mu_{2}}\right)$ such that system (1.3) possesses a least energy solution whenever $\beta \in\left(-\infty, \beta_{0}\right)$. When the potentials $V_{1}(x)$ and $V_{2}(x)$ satisfy:

$(\bar{V}) 0<\inf _{x \in \mathbb{R}^{N}} V_{l}(x)<\lim _{|x| \rightarrow \infty} V_{l}(x) \leq \infty$ for $l=1,2$.

Lin and Wei [32] studied the minimization of the functional $E_{\varepsilon}$ for system (1.3) on $\mathbb{R}^{N}, N=2,3$. When $\beta<0$ and $\varepsilon>0$ sufficiently small, problem (1.3) has a least energy solution $\left(u_{\varepsilon, 1}, u_{\varepsilon, 2}\right)$, such that

$$
\varepsilon^{-N} E_{\varepsilon}\left(u_{\varepsilon, 1}, u_{\varepsilon, 2}\right) \rightarrow \alpha_{\lambda_{1}, \mu_{1}}+\alpha_{\lambda_{2}, \mu_{2}},
$$

as $\varepsilon$ goes to zero (up to a subsequence), where $\alpha_{\lambda_{l}, \mu_{l}}$ is defined by

$$
\begin{gathered}
\alpha_{\lambda_{l}, \mu_{l}}=\inf \left\{I_{\lambda_{l}, \mu_{l}}(u) \mid u \in \mathbf{M}_{\lambda_{l}, \mu_{l}}\right\} \\
I_{\lambda_{l}, \mu_{l}}(u)=\frac{1}{2} \int_{\mathbb{R}^{N}}\left(|\nabla u|^{2}+\lambda_{l} u^{2}\right) \mathrm{d} x-\frac{\mu_{l}}{4} \int_{\mathbb{R}^{N}} u^{4} \mathrm{~d} x, \quad \forall u \in H^{1}\left(\mathbb{R}^{N}\right), \\
\mathbf{M}_{\lambda_{l}, \mu_{l}}=\left\{u \in H^{1}\left(\mathbb{R}^{N}\right) \backslash\{0\} \mid\left\langle I_{\lambda_{l}, \mu_{l}}^{\prime}(u), u\right\rangle=0\right\}
\end{gathered}
$$

for $l=1,2$. Furthermore, if $V_{1}(x)$ and $V_{2}(x)$ are of $C^{2}$ functions with nondegenerate minimum points at $z_{1}$ and $z_{2}$, respectively, then $u_{\varepsilon, l}$ has only one maximum point $z_{l}^{\varepsilon}$ that satisfies

$$
\begin{aligned}
V_{l}\left(z_{l}^{\varepsilon}\right) & \rightarrow \inf _{x \in \mathbb{R}^{N}} V_{l}(x), \quad l=1,2, \\
U_{\epsilon, l}(y) & =u_{\varepsilon, l}\left(z_{l}^{\epsilon}+\epsilon y\right) \rightarrow \omega_{\lambda_{l}, \mu_{l}}(y), \quad l=1,2, \\
\frac{\left|z_{1}^{\varepsilon}-z_{2}^{\varepsilon}\right|}{\varepsilon} & \rightarrow+\infty
\end{aligned}
$$


as $\varepsilon \rightarrow 0$ (up to a subsequence), where $\omega_{\lambda_{l}, \mu_{l}}(x)=\omega_{\lambda_{l}, \mu_{l}}(|x|)$ is the energy minimizer of the minimum $\alpha_{\lambda_{l}, \mu_{l}}>0$ (cf. [43]) and is the unique solution (cf. [27]) of

$$
-\Delta u+\lambda_{l} u=\mu_{l} u^{3} \text { in } \mathbb{R}^{N}, \quad u>0 \text { in } \mathbb{R}^{N} . \quad\left(E_{\lambda_{l}, \mu_{l}}\right)
$$

Consequently, the $u_{\varepsilon, l}$ 's satisfy

$$
\varepsilon^{-N} \int_{B^{N}\left(z_{l}^{\varepsilon} ; \varepsilon R\right)} u_{\varepsilon, l}^{4} \geq d>0
$$

as $\varepsilon \rightarrow 0$ (up to a subsequence), where $d$ and $R$ are positive constants independent of $\varepsilon, B^{N}\left(z_{l}^{\varepsilon} ; \varepsilon R\right)$ is an $N$ dimensional ball with a radius $\varepsilon R$ and a center at $z_{l}^{\varepsilon}$. Hereafter, the point $z_{l}^{\varepsilon}$ is defined as a concentration point of $u_{\varepsilon, l}$ if and only if (1.4) holds.

In the nonlocal case, that is, when $\alpha \in(0,1)$, even in the power type nonlinearities case, there are very few results for the fractional Laplacian systems. In [23], Guo and He studied the following fractional Schrödinger system with nonconstant potentials:

$$
\begin{cases}\varepsilon^{2 \alpha}(-\Delta)^{\alpha} u+P_{1}(x) u=\left(|u|^{2 p}+\beta|u|^{p-1}|v|^{p+1}\right) u, & \text { in } \mathbb{R}^{N} \\ \varepsilon^{2 \alpha}(-\Delta)^{\alpha} v+P_{2}(x) v=\left(|v|^{2 p}+\beta|v|^{p-1}|u|^{p+1}\right) v, & \text { in } \mathbb{R}^{N}\end{cases}
$$

where $\alpha \in(0,1), 0<p<\frac{2 \alpha}{N-2 \alpha}, \varepsilon>0$ is a small parameter and coupling function $\beta \equiv b>0$. Under some appropriate hypotheses on the potentials $P_{1}(x)$ and $P_{2}(x)$, the authors obtained the existence of nontrivial nonnegative solutions for system (1.5) which concentrate around local minima of the potentials. When $\varepsilon=1, P_{1}(x)=1$ and $P_{2}(x)=\omega^{2 \alpha}, \omega>0$, Guo and He [24] obtained the existence of a least energy solution for system (1.5) on the Nehari manifold. Furthermore, the existence of least energy positive solution with both nontrivial components was also established. In [37, Lü and Peng studied system (1.5) with $P_{1}(x)=P_{2}(x)=\varepsilon=1, \quad\left(|u|^{2 p}+\beta|u|^{p-1}|v|^{p+1}\right) u$ and $\left(|v|^{2 p}+\beta|v|^{p-1}|u|^{p+1}\right) v$ being replaced by $f(u)+\beta v$ and $g(v)+\beta u$, respectively. Under very weak assumptions on the nonlinear terms $f$ and $g$, they obtained the existence of positive vector solutions and vector ground state solutions for system (1.5). Moreover, the asymptotic behavior of the solutions as $\beta \rightarrow 0$ was also analyzed by them. For the other related results about the fractional Laplacian system, we refer the readers to [8, 9, 12] and the references therein.

Motivated by [23, 31, 32, 33, 37, it is very natural for us to pose some questions, in particular, such as:

(I) As pointed out in [23, 37, when the coupled nonlinear terms are replaced by $\beta(x)|u|^{q-1} u|v|^{q+1}$ and $\beta(x)|u|^{q+1}|v|^{q-1} v$ for the coupling function $\beta<0$ in $\mathbb{R}^{N}$ and $0<q \leq p$, is the existence of positive solutions for system (1.5) which concentrate around local minima of the potentials still true?

(II) Can the relationship of the minimum points of the potentials $V_{1}(x)$ and $V_{2}(x)$ affects the number of positive solutions for system $\left(P_{\varepsilon}\right)$ ?

$(I I I)$ Under our assumptions $\left(D_{1}\right)$ and $\left(D_{2}\right)$, can one prove that the positive solution of problem $\left(P_{\varepsilon}\right)$ is a least energy solution? If not, can one give some appropriate conditions on the potentials to assure that the positive solution is a least energy solution of problem $\left(P_{\varepsilon}\right)$ ?

In the present paper, by using the Nehari manifold technique, the energy estimates and the Lusternik-Schnirelmann theory of critical points, we study how the relationship of the minimum points of the potentials $V_{1}(x)$ and $V_{2}(x)$ affects the number of positive solutions for system $\left(P_{\varepsilon}\right)$, provided $\beta$ is a nonpositive, bounded and uniformly continuous function on $\mathbb{R}^{N}$, and will give answers to Questions $(I)-(I I I)$. Moreover, whether the positive solution for problem $\left(P_{\varepsilon}\right)$ is a least energy solution depends on the relationship between $\liminf _{|x| \rightarrow \infty} V_{l}(x), \lim _{|x| \rightarrow \infty} V_{l}(x)$ and $\lambda_{l}, l=1,2$.

Before we describe the main results, we need some known techniques. The energy functional $J_{\varepsilon}$ 
we consider that corresponds to problem $\left(P_{\varepsilon}\right)$ is given by, for each $(u, v) \in H=H^{\alpha}\left(\mathbb{R}^{N}\right) \times H^{\alpha}\left(\mathbb{R}^{N}\right)$,

$$
\begin{aligned}
J_{\varepsilon}(u, v)= & \frac{1}{2}\|(u, v)\|_{H}^{2}-\frac{1}{2 p+2}\left(\int_{\mathbb{R}^{N}} \mu_{1}\left|u^{+}\right|^{2 p+2} \mathrm{~d} x+\int_{\mathbb{R}^{N}} \mu_{2}\left|v^{+}\right|^{2 p+2} \mathrm{~d} x\right) \\
& -\frac{1}{q+1} \int_{\mathbb{R}^{N}} \beta(x)\left|u^{+}\right|^{q+1}\left|v^{+}\right|^{q+1} \mathrm{~d} x,
\end{aligned}
$$

where with $u^{+}=\max \{u, 0\}, v^{+}=\max \{v, 0\}$ and the norm $\|\cdot\|_{H}$, given by

$$
\begin{aligned}
\|(u, v)\|_{H}^{2} & =\|u\|_{V_{1}}^{2}+\|v\|_{V_{2}}^{2} \\
& =\int_{\mathbb{R}^{N}}\left(\varepsilon^{2 \alpha}\left|(-\triangle)^{\frac{\alpha}{2}} u\right|^{2}+V_{1}(x) u^{2}\right) \mathrm{d} x+\int_{\mathbb{R}^{N}}\left(\varepsilon^{2 \alpha}\left|(-\triangle)^{\frac{\alpha}{2}} v\right|^{2}+V_{2}(x) v^{2}\right) \mathrm{d} x .
\end{aligned}
$$

Note that the fractional Sobolev space $H^{\alpha}\left(\mathbb{R}^{N}\right)$ is given by:

$$
H^{\alpha}\left(\mathbb{R}^{N}\right)=\left\{u \in L^{2}\left(\mathbb{R}^{N}\right): \int_{\mathbb{R}^{N}} \int_{\mathbb{R}^{N}} \frac{|u(x)-u(y)|^{2}}{|x-y|^{N+2 \alpha}} \mathrm{d} x \mathrm{~d} y<+\infty\right\} .
$$

From Proposition 3.4, 3.6 of [13], we know that

$$
\int_{\mathbb{R}^{N}} \int_{\mathbb{R}^{N}} \frac{|u(x)-u(y)|^{2}}{|x-y|^{N+2 \alpha}} \mathrm{d} x \mathrm{~d} y=2 C(N, \alpha) \int_{\mathbb{R}^{N}}\left|(-\triangle)^{\frac{\alpha}{2}} u\right|^{2} \mathrm{~d} x,
$$

where

$$
C(N, \alpha)=\left(\int_{\mathbb{R}^{N}} \frac{1-\cos \zeta_{1}}{|\zeta|^{N+2 \alpha}} \mathrm{d} \zeta\right), \quad \zeta=\left(\zeta_{1}, \zeta_{2}, \cdots, \zeta_{N}\right)
$$

It is well known that the energy functional $J_{\varepsilon}$ is of class $C^{1}$ in $H$ and the nonnegative solutions of problem $\left(P_{\varepsilon}\right)$ are the critical points of the energy functional $J_{\varepsilon}$. As the energy functional $J_{\varepsilon}$ is not bounded below on $H$ and to prove the existence of nontrivial critical points of $J_{\varepsilon}$, it is useful to consider the functional on the Nehari manifold

$$
\mathbf{N}_{\varepsilon}=\left\{(u, v) \in \mathbf{T}: \begin{array}{l}
\|u\|_{V_{1}}^{2}=\mu_{1} \int_{\mathbb{R}^{N}}\left|u^{+}\right|^{2 p+2} \mathrm{~d} x+\int_{\mathbb{R}^{N}} \beta(x)\left|u^{+}\right|^{q+1}\left|v^{+}\right|^{q+1} \mathrm{~d} x, \\
\|v\|_{V_{2}}^{2}=\mu_{2} \int_{\mathbb{R}^{N}}\left|v^{+}\right|^{2 p+2} \mathrm{~d} x+\int_{\mathbb{R}^{N}} \beta(x)\left|u^{+}\right|^{q+1}\left|v^{+}\right|^{q+1} \mathrm{~d} x
\end{array}\right\},
$$

where

$$
\mathbf{T}=\{(u, v) \in H: u \not \equiv 0 \text { and } v \not \equiv 0\} .
$$

Furthermore, we consider the minimization problem:

$$
c_{\varepsilon}=\inf \left\{J_{\varepsilon}(u, v):(u, v) \in \mathbf{N}_{\varepsilon}\right\},
$$

we call the nontrivial critical point $(u, v) \in H$ of $J_{\varepsilon}$ is a least energy solution of problem $\left(P_{\varepsilon}\right)$ if $J_{\varepsilon}(u, v)=c_{\varepsilon}$ and $(u, v) \in \mathbf{N}_{\varepsilon}$. Note that if there exists a nontrivial solution $(u, v) \in \mathbf{N}_{\varepsilon}$ of problem $\left(P_{\varepsilon}\right)$ such that $J_{\varepsilon}(u, v)>c_{\varepsilon}$, then we call the solution $(u, v)$ is a higher energy solution of problem $\left(P_{\varepsilon}\right)$.

Now we state our main results.

Theorem $1.2(i)$ Assume that the conditions $\left(D_{1}\right)-\left(D_{4}\right)$ hold. Then there exists $\varepsilon_{0}>0$ such that for every $\varepsilon \in\left(0, \varepsilon_{0}\right)$, problem $\left(P_{\varepsilon}\right)$ has at least $k \times \ell+m$ positive solutions.

(ii) Let $\left(\widehat{u}_{\varepsilon, i}, \widehat{v}_{\varepsilon, j}\right)$ be a positive solution of problem $\left(P_{\varepsilon}\right)$ as in part $(i)$. Then there exist $z_{1, i}$ and $z_{2, j}$ which are isolated gobal minimum points of potentials $V_{1}(x)$ and $V_{2}(x)$, respectively such that $\left(\widehat{u}_{\varepsilon, i}, \widehat{v}_{\varepsilon, j}\right)$ concentrating at $\left(z_{1, i}, z_{2, j}\right)$ as $\varepsilon \rightarrow 0$. 
Theorem 1.3 (i) If $\liminf _{|x| \rightarrow \infty} V_{l}(x) \equiv V_{l, \infty}>\lambda_{l}$ for all $l=1,2$, then there exists $0<\varepsilon_{* *} \leq \varepsilon_{0}$ such that for every $\varepsilon<\varepsilon_{* *}$, we can find at least one least energy solution in the these solutions of Theorem 1.2 $(i)$.

(ii) If $\lim _{|x| \rightarrow \infty} V_{l}(x)=\lambda_{l}$ for all $l=1,2$, then all of the solutions of Theorem 1.2 $(i)$ are higher energy.

Remark 1.1 Compared with the local operator $-\Delta$, the operator $(-\Delta)^{\alpha}$ with $\alpha \in(0,1)$ on $\mathbb{R}^{N}$ is nonlocal, which can be expressed as follows: the quantity $(-\Delta)^{\alpha} u(x)$ depend on not only the values of $u$ in a neighborhood of $x$ (as is the case for the Laplacian), but also the values of $u$ at any point $y \in \mathbb{R}^{N}$, and it is expected that the standard techniques for $-\Delta$ cannot be used directly.

Remark 1.2 The main difficulty when dealing with problem $\left(P_{\varepsilon}\right)$ lies in the lack of compactness of the embedding from $H^{\alpha}\left(\mathbb{R}^{N}\right)$ into $L^{r}\left(\mathbb{R}^{N}\right), 2 \leq r<2_{\alpha}^{*}$, which prevents us from using the variational methods in a standard way. We solve this difficulty by using the Nehari manifold technique and the energy estimates, see Section 3 for details.

Notation 1.1 Throughout this paper, we shall denote $|\cdot|_{r}$ the $L^{r}$-norm for $1 \leq r \leq+\infty$ and $C$ various positive generic constants, which may vary from line to line. $B^{N}(x, r)$ denotes a ball centered at $x$ with radius $r$ in $\mathbb{R}^{N}$. Also if we take a subsequence of a sequence $\left\{\left(u_{n}, v_{n}\right)\right\}$ we shall denote it again by $\left\{\left(u_{n}, v_{n}\right)\right\}$. We use o(1) to denote any quantity which tends to zero as $n \rightarrow \infty$ and $o_{\varepsilon}(1)$ to denote any quantity which tends to zero as $\varepsilon \rightarrow 0$.

The remainder of this paper is as follows. In Section 2, we give some preliminaries. In Section 3, we construct the Palais-Smale (PS) sequences. In Section 4, we prove Theorem 1.2, In Section 5, we prove Theorem 1.3 .

\section{Preliminaries}

First of all, it is easy to see that if we make the change of variables $x=\varepsilon z$, then we can rewrite Eq. $\left(P_{\varepsilon}\right)$ as the following equivalent equation:

$$
\begin{cases}(-\Delta)^{\alpha} u+V_{1}(\varepsilon x) u=\left(\mu_{1}|u|^{2 p}+\beta(\varepsilon x)|u|^{q-1}|v|^{q+1}\right) u, & \text { in } \mathbb{R}^{N} \\ (-\Delta)^{\alpha} v+V_{2}(\varepsilon x) v=\left(\mu_{2}|v|^{2 p}+\beta(\varepsilon x)|v|^{q-1}|u|^{q+1}\right) v, & \text { in } \mathbb{R}^{N} \\ u, v \in H^{\alpha}\left(\mathbb{R}^{N}\right), \quad u, v>0, & \text { in } \mathbb{R}^{N}\end{cases}
$$

Now we present some related results about Eq. $\left(\widehat{E}_{l}\right)$. It is obvious that Eq. $\left(\widehat{E}_{l}\right)$ is variational, and its solutions are the critical points of the functional $\widehat{I}_{l}(u)$ defined in $H^{\alpha}\left(\mathbb{R}^{N}\right)$ as

$$
\widehat{I}_{l}(u)=\frac{1}{2} \int_{\mathbb{R}^{N}}\left(\left|(-\triangle)^{\frac{\alpha}{2}} u\right|^{2}+\lambda_{l} u^{2}\right) \mathrm{d} x-\frac{\mu_{l}}{2 p+2} \int_{\mathbb{R}^{N}}\left|u^{+}\right|^{2 p+2} \mathrm{~d} x, \quad l=1,2 .
$$

Furthermore, one can see that $\widehat{I}_{l}$ is a $C^{1}$ functional with the derivative given by

$$
\left\langle\widehat{I}_{l}^{\prime}(u), \varphi\right\rangle=\int_{\mathbb{R}^{N}}\left((-\triangle)^{\frac{\alpha}{2}} u(-\triangle)^{\frac{\alpha}{2}} v+\lambda_{l} u \varphi\right) \mathrm{d} x-\mu_{l} \int_{\mathbb{R}^{N}}\left|u^{+}\right|^{2 p} u^{+} \varphi \mathrm{d} x, \quad l=1,2,
$$

for all $\varphi \in H^{\alpha}\left(\mathbb{R}^{N}\right)$, where $\widehat{I}_{l}^{\prime}$ denotes the Fréchet derivative of $\widehat{I}_{l}$.

Define the Nehari manifold

$$
\widehat{\mathbf{M}}_{l}:=\left\{u \in H^{\alpha}\left(\mathbb{R}^{N}\right) \backslash\{0\}:\left\langle\widehat{I}_{l}^{\prime}(u), u\right\rangle=0\right\} \text { for } l=1,2 .
$$


Then, by Frank, Lenzmann and Silvestre [20], we may assume that Eq. $\left(\widehat{E}_{l}\right)$ has a unique least energy positive solution $\widehat{\omega}_{l}$ (which up to translation) such that

$$
\widehat{\alpha}_{l}=\inf _{u \in \widehat{\mathbf{M}}_{l}} \widehat{I}_{l}(u)=\widehat{I}_{l}\left(\widehat{\omega}_{l}\right) \text { for } l=1,2 .
$$

Furthermore, $\widehat{\omega}_{l}$ is radial, i.e., $\widehat{\omega}_{l}(x)=\widehat{\omega}_{l}(|x|)$.

To prove the main results, we will show some technical lemmas, whose proofs follow with the same type of arguments found in [31. However for the readers' convenience we will write their proofs. we need the following lemmas.

Lemma 2.1 Assume that $\varepsilon>0$ and $(u, v) \in \mathbf{N}_{\varepsilon}$. Then

$$
\mu_{1} \int_{\mathbb{R}^{N}}\left|u^{+}\right|^{2 p+2} \mathrm{~d} x \geq \int_{\mathbb{R}^{N}}\left(\varepsilon^{2 \alpha}\left|(-\triangle)^{\frac{\alpha}{2}} u\right|^{2}+V_{1}(x) u^{2}\right) \mathrm{d} x \geq \frac{2 p+2}{p} \varepsilon^{N} \widehat{\alpha}_{1}
$$

and

$$
\mu_{2} \int_{\mathbb{R}^{N}}\left|v^{+}\right|^{2 p+2} \mathrm{~d} x \geq \int_{\mathbb{R}^{N}}\left(\varepsilon^{2 \alpha}\left|(-\triangle)^{\frac{\alpha}{2}} v\right|^{2}+V_{2}(x) v^{2}\right) \mathrm{d} x \geq \frac{2 p+2}{p} \varepsilon^{N} \widehat{\alpha}_{2} .
$$

Furthermore, if $\int_{\mathbb{R}^{N}} \beta(x)\left|u^{+}\right|^{q+1}\left|v^{+}\right|^{q+1} \mathrm{~d} x<0$, then all the inequalities of (2.1) and (2.2) become strict, i.e.

$$
\mu_{1} \int_{\mathbb{R}^{N}}\left|u^{+}\right|^{2 p+2} \mathrm{~d} x>\int_{\mathbb{R}^{N}}\left(\varepsilon^{2 \alpha}\left|(-\triangle)^{\frac{\alpha}{2}} u\right|^{2}+V_{1}(x) u^{2}\right) \mathrm{d} x>\frac{2 p+2}{p} \varepsilon^{N} \widehat{\alpha}_{1}
$$

and

$$
\mu_{2} \int_{\mathbb{R}^{N}}\left|v^{+}\right|^{2 p+2} \mathrm{~d} x>\int_{\mathbb{R}^{N}}\left(\varepsilon^{2 \alpha}\left|(-\triangle)^{\frac{\alpha}{2}} v\right|^{2}+V_{2}(x) v^{2}\right) \mathrm{d} x>\frac{2 p+2}{p} \varepsilon^{N} \widehat{\alpha}_{2}
$$

Proof. Let $(u, v) \in \mathbf{N}_{\varepsilon}$. Set $u_{\varepsilon}(x)=u(\varepsilon x)$ and $v_{\varepsilon}(x)=v(\varepsilon x)$ for $x \in \mathbb{R}^{N}$. Due to $\beta(x) \leq 0$ in $\mathbb{R}^{N}$ and $(u, v) \in \mathbf{N}_{\varepsilon}$, it is obvious that

$$
\begin{aligned}
\int_{\mathbb{R}^{N}}\left(\left|(-\triangle)^{\frac{\alpha}{2}} u_{\varepsilon}\right|^{2}+\lambda_{1} u_{\varepsilon}^{2}\right) \mathrm{d} x & \leq \int_{\mathbb{R}^{N}}\left(\left|(-\triangle)^{\frac{\alpha}{2}} u_{\varepsilon}\right|^{2}+V_{1}(\varepsilon x) u_{\varepsilon}^{2}\right) \mathrm{d} x \\
& =\mu_{1} \int_{\mathbb{R}^{N}}\left|u_{\varepsilon}^{+}\right|^{2 p+2} \mathrm{~d} x+\int_{\mathbb{R}^{N}} \beta(\varepsilon x)\left|u_{\varepsilon}^{+}\right|^{q+1}\left|v_{\varepsilon}^{+}\right|^{q+1} \mathrm{~d} x \\
& \leq \mu_{1} \int_{\mathbb{R}^{N}}\left|u_{\varepsilon}^{+}\right|^{2 p+2} \mathrm{~d} x
\end{aligned}
$$

and

$$
\begin{aligned}
\int_{\mathbb{R}^{N}}\left(\left|(-\triangle)^{\frac{\alpha}{2}} v_{\varepsilon}\right|^{2}+\lambda_{2} v_{\varepsilon}^{2}\right) \mathrm{d} x & \leq \int_{\mathbb{R}^{N}}\left(\left|(-\triangle)^{\frac{\alpha}{2}} v_{\varepsilon}\right|^{2}+V_{2}(\varepsilon x) v_{\varepsilon}^{2}\right) \mathrm{d} x \\
& =\mu_{2} \int_{\mathbb{R}^{N}}\left|v_{\varepsilon}^{+}\right|^{2 p+2} \mathrm{~d} x+\int_{\mathbb{R}^{N}} \beta(\varepsilon x)\left|u_{\varepsilon}^{+}\right|^{q+1}\left|v_{\varepsilon}^{+}\right|^{q+1} \mathrm{~d} x \\
& \leq \mu_{2} \int_{\mathbb{R}^{N}}\left|v_{\varepsilon}^{+}\right|^{2 p+2} \mathrm{~d} x .
\end{aligned}
$$

Let

$$
s_{\varepsilon}=\left(\frac{\int_{\mathbb{R}^{N}}\left(\left|(-\triangle)^{\frac{\alpha}{2}} u_{\varepsilon}\right|^{2}+\lambda_{1} u_{\varepsilon}^{2}\right) \mathrm{d} x}{\mu_{1} \int_{\mathbb{R}^{N}}\left|u_{\varepsilon}^{+}\right|^{2 p+2} \mathrm{~d} x}\right)^{1 / 2 p} \text { and } t_{\varepsilon}=\left(\frac{\int_{\mathbb{R}^{N}}\left(\left|(-\triangle)^{\frac{\alpha}{2}} v_{\varepsilon}\right|^{2}+\lambda_{2} v_{\varepsilon}^{2}\right) \mathrm{d} x}{\mu_{2} \int_{\mathbb{R}^{N}}\left|v_{\varepsilon}^{+}\right|^{2 p+2} \mathrm{~d} x}\right)^{1 / 2 p} .
$$


Then by (2.3) and (2.4), it is easy to verify that $0<s_{\varepsilon}, t_{\varepsilon} \leq 1, s_{\varepsilon} u_{\varepsilon} \in \widehat{\mathbf{M}}_{1}$ and $t_{\varepsilon} v_{\varepsilon} \in \widehat{\mathbf{M}}_{2}$. Hence,

$$
\widehat{\alpha}_{1} \leq \widehat{I}_{1}\left(s_{\varepsilon} u_{\varepsilon}\right) \leq \frac{p}{2 p+2} \int_{\mathbb{R}^{N}}\left(\left|(-\triangle)^{\frac{\alpha}{2}} u_{\varepsilon}\right|^{2}+\lambda_{1} u_{\varepsilon}^{2}\right) \mathrm{d} x
$$

and

$$
\widehat{\alpha}_{2} \leq \widehat{I}_{2}\left(t_{\varepsilon} v_{\varepsilon}\right) \leq \frac{p}{2 p+2} \int_{\mathbb{R}^{N}}\left(\left|(-\triangle)^{\frac{\alpha}{2}} v_{\varepsilon}\right|^{2}+\lambda_{2} v_{\varepsilon}^{2}\right) \mathrm{d} x .
$$

Thus by (2.3) - (2.6), we obtain (2.1) and (2.2). Similarly, one may follow the above argument to show that all the inequalities of (2.1) and (2.2) become strict if $\int_{\mathbb{R}^{N}} \beta(x)\left|u^{+}\right|^{q+1}\left|v^{+}\right|^{q+1} \mathrm{~d} x<0$. Therefore, we may complete the proof.

The integral $\int_{\mathbb{R}^{N}} \beta(x)\left|u^{+}\right|^{q+1}\left|v^{+}\right|^{q+1} \mathrm{~d} x$ may play an important role in the quantity of the energy functional $J_{\varepsilon}$. Here we state the crucial energy estimates, given by

Lemma 2.2 Let $\varepsilon, \sigma>0$ and $(u, v) \in \mathbf{N}_{\varepsilon}$. If $\int_{\mathbb{R}^{N}} \beta(x)\left|u^{+}\right|^{q+1}\left|v^{+}\right|^{q+1} \mathrm{~d} x \leq-\sigma \varepsilon^{N}$, then

$$
\varepsilon^{-N} J_{\varepsilon}(u, v)>\widehat{\alpha}_{1}+\widehat{\alpha}_{2}+\bar{\delta}
$$

where

$$
\bar{\delta}=\frac{p \sigma}{p+1} \min \left\{1, \frac{1}{2} \min \left\{\frac{S_{1}^{p+1}}{\mu_{1}\left(\frac{2 p+2}{p} \widehat{\alpha}_{1}+\sigma\right)^{p}}, \frac{S_{2}^{p+1}}{\mu_{2}\left(\frac{2 p+2}{p} \widehat{\alpha}_{2}+\sigma\right)^{p}}\right\}\right\},
$$

and

$$
S_{l}=\inf _{w \in H^{\alpha}\left(\mathbb{R}^{N}\right) \backslash\{0\}} \frac{\int_{\mathbb{R}^{N}}\left(\left|(-\triangle)^{\frac{\alpha}{2}} w\right|^{2}+\lambda_{l} w^{2}\right) \mathrm{d} x}{\left(\int_{\mathbb{R}^{N}}\left|w^{+}\right|^{2 p+2} \mathrm{~d} x\right)^{1 / p+1}}>0, \quad l=1,2 .
$$

Furthermore, if $0<\sigma<(2 p+2) \sqrt{\widehat{\alpha}_{1} \widehat{\alpha}_{2}}$, then

$$
\begin{aligned}
& p^{2} \mu_{1} \mu_{2} \int_{\mathbb{R}^{N}}\left|u^{+}\right|^{2 p+2} \mathrm{~d} x \int_{\mathbb{R}^{N}}\left|v^{+}\right|^{2 p+2} \mathrm{~d} x-\left(\int_{\mathbb{R}^{N}} \beta(x)\left|u^{+}\right|^{q+1}\left|v^{+}\right|^{q+1} \mathrm{~d} x\right)^{2} \\
> & \varepsilon^{2 N}\left(4(p+1)^{2} \widehat{\alpha}_{1} \widehat{\alpha}_{2}-\sigma^{2}\right)>0
\end{aligned}
$$

for all $(u, v) \in \mathbf{N}_{\varepsilon}$ and $\varepsilon^{-N} J_{\varepsilon}(u, v) \leq \widehat{\alpha}_{1}+\widehat{\alpha}_{2}+\bar{\delta}$.

Proof. Assume $(u, v) \in \mathbf{N}_{\varepsilon}$ and $\sigma>0$ such that

$$
\int_{\mathbb{R}^{N}} \beta(x)\left|u^{+}\right|^{q+1}\left|v^{+}\right|^{q+1} \mathrm{~d} x \leq-\sigma \varepsilon^{N} .
$$

Let $\widetilde{u}(x)=u(\varepsilon x)$ and $\widetilde{v}(x)=v(\varepsilon x)$. Then

$$
\varepsilon^{-N} J_{\varepsilon}(u, v)=\widetilde{J}_{\varepsilon}(\widetilde{u}, \widetilde{v}),
$$

where

$$
\begin{aligned}
\widetilde{J}_{\varepsilon}(\widetilde{u}, \widetilde{v})= & \frac{1}{2} \int_{\mathbb{R}^{N}}\left(\left|(-\triangle)^{\frac{\alpha}{2}} \widetilde{u}\right|^{2}+V_{1}(\varepsilon x) \widetilde{u}^{2}\right) \mathrm{d} x+\frac{1}{2} \int_{\mathbb{R}^{N}}\left(\left|(-\triangle)^{\frac{\alpha}{2}} \widetilde{v}\right|^{2}+V_{2}(\varepsilon x) \widetilde{v}^{2}\right) \mathrm{d} x \\
& -\frac{1}{2 p+2}\left(\int_{\mathbb{R}^{N}} \mu_{1}\left|\widetilde{u}^{+}\right|^{2 p+2}+\int_{\mathbb{R}^{N}} \mu_{2}\left|\widetilde{v}^{+}\right|^{2 p+2}\right) \mathrm{d} x \\
& -\frac{1}{q+1} \int_{\mathbb{R}^{N}} \beta(\varepsilon x)\left|\widetilde{u}^{+}\right|^{q+1}\left|\widetilde{v}^{+}\right|^{q+1} \mathrm{~d} x .
\end{aligned}
$$


Hence, by (2.11), (2.12) and $(u, v) \in \mathbf{N}_{\varepsilon}$, we have

$$
\begin{aligned}
& \int_{\mathbb{R}^{N}} \beta(\varepsilon x)\left|\widetilde{u}^{+}\right|^{q+1}\left|\widetilde{v}^{+}\right|^{q+1} \mathrm{~d} x \leq-\sigma \\
& \int_{\mathbb{R}^{N}}\left(\left|(-\triangle)^{\frac{\alpha}{2}} \widetilde{u}\right|^{2}+\lambda_{1} \widetilde{u}^{2}\right) \mathrm{d} x \leq \int_{\mathbb{R}^{N}}\left(\left|(-\triangle)^{\frac{\alpha}{2}} \widetilde{u}\right|^{2}+V_{1}(\varepsilon x) \widetilde{u}^{2}\right) \mathrm{d} x \\
&=\mu_{1} \int_{\mathbb{R}^{N}}\left|\widetilde{u}^{+}\right|^{2 p+2} \mathrm{~d} x+\int_{\mathbb{R}^{N}} \beta(\varepsilon x)\left|\widetilde{u}^{+}\right|^{q+1}\left|\widetilde{v}^{+}\right|^{q+1} \mathrm{~d} x
\end{aligned}
$$

and

$$
\begin{aligned}
\int_{\mathbb{R}^{N}}\left(\left|(-\triangle)^{\frac{\alpha}{2}} \widetilde{v}\right|^{2}+\lambda_{2} \widetilde{v}^{2}\right) \mathrm{d} x & \leq \int_{\mathbb{R}^{N}}\left(\left|(-\triangle)^{\frac{\alpha}{2}} \widetilde{v}\right|^{2}+V_{2}(\varepsilon x) \widetilde{v}^{2}\right) \mathrm{d} x \\
& =\mu_{2} \int_{\mathbb{R}^{N}}\left|\widetilde{v}^{+}\right|^{2 p+2} \mathrm{~d} x+\int_{\mathbb{R}^{N}} \beta(\varepsilon x)\left|\widetilde{u}^{+}\right|^{q+1}\left|\widetilde{v}^{+}\right|^{q+1} \mathrm{~d} x
\end{aligned}
$$

Moreover, it follows from (2.12), (2.14) and (2.15) that

$$
\begin{aligned}
\widetilde{J}_{\varepsilon}(\widetilde{u}, \widetilde{v}) \geq & \frac{1}{2} \int_{\mathbb{R}^{N}}\left(\left|(-\triangle)^{\frac{\alpha}{2}} \widetilde{u}\right|^{2}+V_{1}(\varepsilon x) \widetilde{u}^{2}\right) \mathrm{d} x+\frac{1}{2} \int_{\mathbb{R}^{N}}\left(\left|(-\triangle)^{\frac{\alpha}{2}} \widetilde{v}\right|^{2}+V_{2}(\varepsilon x) \widetilde{v}^{2}\right) \mathrm{d} x \\
& -\frac{1}{2 p+2} \int_{\mathbb{R}^{N}} \mu_{1}\left|\widetilde{u}^{+}\right|^{2 p+2}-\frac{1}{2 p+2} \int_{\mathbb{R}^{N}} \mu_{2}\left|\widetilde{v}^{+}\right|^{2 p+2} \mathrm{~d} x \\
& -\frac{1}{p+1} \int_{\mathbb{R}^{N}} \beta(\varepsilon x)\left|\widetilde{u}^{+}\right|^{q+1}\left|\widetilde{v}^{+}\right|^{q+1} \mathrm{~d} x \\
= & \frac{p}{2 p+2}\left[\int_{\mathbb{R}^{N}}\left(\left|(-\triangle)^{\frac{\alpha}{2}}\right|^{2}+V_{1}(\varepsilon x) \widetilde{u}^{2}\right) \mathrm{d} x+\int_{\mathbb{R}^{N}}\left(\left|(-\triangle)^{\frac{\alpha}{2}} \widetilde{v}\right|^{2}+V_{2}(\varepsilon x) \widetilde{v}^{2}\right) \mathrm{d} x\right] .
\end{aligned}
$$

Set

$$
\widetilde{s}=\left(\frac{\int_{\mathbb{R}^{N}}\left(\left|(-\triangle)^{\frac{\alpha}{2}} \widetilde{u}\right|^{2}+\lambda_{1} \widetilde{u}^{2}\right) \mathrm{d} x}{\mu_{1} \int_{\mathbb{R}^{N}}\left|\widetilde{u}^{+}\right|^{2 p+2} \mathrm{~d} x}\right)^{1 / 2 p} \text { and } \tilde{t}=\left(\frac{\int_{\mathbb{R}^{N}}\left(\left|(-\triangle)^{\frac{\alpha}{2}} \widetilde{v}\right|^{2}+\lambda_{2} \widetilde{v}^{2}\right) \mathrm{d} x}{\mu_{2} \int_{\mathbb{R}^{N}}\left|\widetilde{v}^{+}\right|^{2 p+2} \mathrm{~d} x}\right)^{1 / 2 p} .
$$

Then, by (2.13) - (2.15), we have

$$
\widetilde{s}^{2 p}=\frac{\int_{\mathbb{R}^{N}}\left(\left|(-\triangle)^{\frac{\alpha}{2}} \widetilde{u}\right|^{2}+\lambda_{1} \widetilde{u}^{2}\right) \mathrm{d} x}{\mu_{1} \int_{\mathbb{R}^{N}}\left|\widetilde{u}^{+}\right|^{2 p+2} \mathrm{~d} x} \leq 1+\frac{\int_{\mathbb{R}^{N}} \beta(\varepsilon x)\left|\widetilde{u}^{+}\right|^{q+1}\left|\widetilde{v}^{+}\right|^{q+1} \mathrm{~d} x}{\mu_{1} \int_{\mathbb{R}^{N}}\left|\widetilde{u}^{+}\right|^{2 p+2} \mathrm{~d} x} \leq 1-\frac{\sigma}{\mu_{1} \int_{\mathbb{R}^{N}}\left|\widetilde{u}^{+}\right|^{2 p+2} \mathrm{~d} x}
$$

and

$$
\widetilde{t}^{2 p}=\frac{\int_{\mathbb{R}^{N}}\left(\left|(-\triangle)^{\frac{\alpha}{2}} \widetilde{v}\right|^{2}+\lambda_{2} \widetilde{v}^{2}\right) \mathrm{d} x}{\mu_{2} \int_{\mathbb{R}^{N}}\left|\widetilde{v}^{+}\right|^{2 p+2} \mathrm{~d} x} \leq 1+\frac{\int_{\mathbb{R}^{N}} \beta(\varepsilon x)\left|\widetilde{u}^{+}\right|^{q+1}\left|\widetilde{v}^{+}\right|^{q+1} \mathrm{~d} x}{\mu_{2} \int_{\mathbb{R}^{N}}\left|\widetilde{v}^{+}\right|^{2 p+2} \mathrm{~d} x} \leq 1-\frac{\sigma}{\mu_{2} \int_{\mathbb{R}^{N}}\left|\widetilde{v}^{+}\right|^{2 p+2} \mathrm{~d} x} .
$$

Besides, $\widetilde{s} \widetilde{u} \in \widehat{\mathbf{M}}_{1}$ and $\widetilde{t} \widetilde{v} \in \widehat{\mathbf{M}}_{2}$ for all $\varepsilon>0,0<q \leq p \leq 1$ if $N \leq 4 \alpha$ and $0<q \leq p \leq \frac{2 \alpha}{N-2 \alpha}$ if $N>4 \alpha$. It follows from (2.16), (2.17) and (2.18) that

$$
\begin{aligned}
\widetilde{J}_{\varepsilon}(\widetilde{u}, \widetilde{v}) \geq & \frac{p}{2 p+2}\left[\int_{\mathbb{R}^{N}}\left(\left|(-\triangle)^{\frac{\alpha}{2}} \widetilde{u}\right|^{2}+\lambda_{1} \widetilde{u}^{2}\right) \mathrm{d} x+\int_{\mathbb{R}^{N}}\left(\left|(-\triangle)^{\frac{\alpha}{2}} \widetilde{v}\right|^{2}+\lambda_{2} \widetilde{v}^{2}\right) \mathrm{d} x\right] \\
\geq & \frac{p}{2 p+2}\left[\widetilde{s}^{2 p} \int_{\mathbb{R}^{N}}\left(\left|(-\triangle)^{\frac{\alpha}{2}} \widetilde{u}\right|^{2}+\lambda_{1} \widetilde{u}^{2}\right) \mathrm{d} x+\widetilde{t}^{2 p} \int_{\mathbb{R}^{N}}\left(\mid(-\triangle)^{\frac{\alpha}{2}} \widetilde{v}^{2}+\lambda_{2} \widetilde{v}^{2}\right) \mathrm{d} x\right] \\
& +\frac{p \sigma}{2 p+2}\left[\frac{\int_{\mathbb{R}^{N}}\left(\left|(-\triangle)^{\frac{\alpha}{2}} \widetilde{u}\right|^{2}+\lambda_{1} \widetilde{u}^{2}\right) \mathrm{d} x}{\mu_{1} \int_{\mathbb{R}^{N}}\left|\widetilde{u}^{+}\right|^{2 p+2} \mathrm{~d} x}+\frac{\int_{\mathbb{R}^{N}}\left(\left|(-\triangle)^{\frac{\alpha}{2}} \widetilde{v}\right|^{2}+\lambda_{2} \widetilde{v}^{2}\right) \mathrm{d} x}{\mu_{2} \int_{\mathbb{R}^{N}}\left|\widetilde{v}^{+}\right|^{2 p+2} \mathrm{~d} x}\right]
\end{aligned}
$$




$$
\begin{aligned}
\geq & \frac{p \sigma}{2 p+2}\left[\frac{\int_{\mathbb{R}^{N}}\left(\left|(-\triangle)^{\frac{\alpha}{2}} \widetilde{u}\right|^{2}+\lambda_{1} \widetilde{u}^{2}\right) \mathrm{d} x}{\mu_{1} \int_{\mathbb{R}^{N}}\left|\widetilde{u}^{+}\right|^{2 p+2} \mathrm{~d} x}+\frac{\int_{\mathbb{R}^{N}}\left(\left|(-\triangle)^{\frac{\alpha}{2}} \widetilde{v}\right|^{2}+\lambda_{2} \widetilde{v}^{2}\right) \mathrm{d} x}{\mu_{2} \int_{\mathbb{R}^{N}}\left|\widetilde{v}^{+}\right|^{2 p+2} \mathrm{~d} x}\right] \\
& +\widehat{\alpha}_{1}+\widehat{\alpha}_{2} .
\end{aligned}
$$

Now we wish to claim (2.7). Suppose both

$$
\left(\frac{2 p+2}{p} \widehat{\alpha}_{1}+\sigma\right)^{p} \int_{\mathbb{R}^{N}}\left(\left|(-\triangle)^{\frac{\alpha}{2}} \widetilde{u}\right|^{2}+\lambda_{1} \widetilde{u}^{2}\right) \mathrm{d} x<S_{1}^{p+1} \int_{\mathbb{R}^{N}}\left|\widetilde{u}^{+}\right|^{2 p+2} \mathrm{~d} x
$$

and

$$
\left(\frac{2 p+2}{p} \widehat{\alpha}_{2}+\sigma\right)^{p} \int_{\mathbb{R}^{N}}\left(\left|(-\triangle)^{\frac{\alpha}{2}} \widetilde{v}\right|^{2}+\lambda_{2} \widetilde{v}^{2}\right) \mathrm{d} x<S_{2}^{p+1} \int_{\mathbb{R}^{N}}\left|\widetilde{v}^{+}\right|^{2 p+2} \mathrm{~d} x .
$$

Then by (2.9), we have

$$
\begin{aligned}
& \left(\frac{2 p+2}{p} \widehat{\alpha}_{1}+\sigma\right)^{p} \int_{\mathbb{R}^{N}}\left(\mid(-\triangle)^{\frac{\alpha}{2}} \widetilde{u}^{2}+\lambda_{1} \widetilde{u}^{2}\right) \mathrm{d} x<S_{1}^{p+1} \int_{\mathbb{R}^{N}}\left|\widetilde{u}^{+}\right|^{2 p+2} \mathrm{~d} x \\
\leq & \left(\int_{\mathbb{R}^{N}}\left(\left|(-\triangle)^{\frac{\alpha}{2}} \widetilde{u}\right|^{2}+\lambda_{1} \widetilde{u}^{2}\right) \mathrm{d} x\right)^{p+1}
\end{aligned}
$$

and

$$
\begin{aligned}
& \left(\frac{2 p+2}{p} \widehat{\alpha}_{2}+\sigma\right)^{p} \int_{\mathbb{R}^{N}}\left(\left|(-\triangle)^{\frac{\alpha}{2}} \widetilde{v}\right|^{2}+\lambda_{2} \widetilde{v}^{2}\right) \mathrm{d} x<S_{2}^{p+1} \int_{\mathbb{R}^{N}}\left|\widetilde{v}^{+}\right|^{2 p+2} \mathrm{~d} x \\
\leq & \left(\int_{\mathbb{R}^{N}}\left(\mid(-\triangle)^{\frac{\alpha}{2}} \widetilde{v}^{2}+\lambda_{2} \widetilde{v}^{2}\right) \mathrm{d} x\right)^{p+1} .
\end{aligned}
$$

Hence,

$$
\int_{\mathbb{R}^{N}}\left(\left|(-\triangle)^{\frac{\alpha}{2}} \widetilde{u}\right|^{2}+\lambda_{1} \widetilde{u}^{2}\right) \mathrm{d} x>\frac{2 p+2}{p} \widehat{\alpha}_{1}+\sigma
$$

and

$$
\int_{\mathbb{R}^{N}}\left(\left|(-\triangle)^{\frac{\alpha}{2}} \widetilde{u}\right|^{2}+\lambda_{2} \widetilde{v}^{2}\right) \mathrm{d} x>\frac{2 p+2}{p} \widehat{\alpha}_{2}+\sigma .
$$

Combining (2.16), (2.20) and (2.21), we obtain

$$
\widetilde{J}_{\varepsilon}(\widetilde{u}, \widetilde{v})>\widehat{\alpha}_{1}+\widehat{\alpha}_{2}+\frac{p \sigma}{p+1},
$$

thus (2.7) holds. On the other hand, suppose either

$$
\left(\frac{2 p+2}{p} \widehat{\alpha}_{1}+\sigma\right)^{p} \int_{\mathbb{R}^{N}}\left(\left|(-\triangle)^{\frac{\alpha}{2}} \widetilde{u}\right|^{2}+\lambda_{1} \widetilde{u}^{2}\right) \mathrm{d} x \geq S_{1}^{p+1} \int_{\mathbb{R}^{N}}\left|\widetilde{u}^{+}\right|^{2 p+2} \mathrm{~d} x
$$

or

$$
\left(\frac{2 p+2}{p} \widehat{\alpha}_{1}+\sigma\right)^{p} \int_{\mathbb{R}^{N}}\left(\left|(-\triangle)^{\frac{\alpha}{2}} \widetilde{v}\right|^{2}+\lambda_{2} \widetilde{v}^{2}\right) \mathrm{d} x \geq S_{2}^{p+1} \int_{\mathbb{R}^{N}}\left|\widetilde{v}^{+}\right|^{2 p+2} \mathrm{~d} x .
$$

Then by (2.19), we obtain

$$
\begin{aligned}
\widetilde{J}_{\varepsilon}(\widetilde{u}, \widetilde{v}) \geq & \frac{p \sigma}{2 p+2}\left[\frac{\int_{\mathbb{R}^{N}}\left(\left|(-\triangle)^{\frac{\alpha}{2}} \widetilde{u}\right|^{2}+\lambda_{1} \widetilde{u}^{2}\right) \mathrm{d} x}{\mu_{1} \int_{\mathbb{R}^{N}}\left|\widetilde{u}^{+}\right|^{2 p+2} \mathrm{~d} x}+\frac{\int_{\mathbb{R}^{N}}\left(\left|(-\triangle)^{\frac{\alpha}{2}} \widetilde{v}\right|^{2}+\lambda_{2} \widetilde{v}^{2}\right) \mathrm{d} x}{\mu_{2} \int_{\mathbb{R}^{N}}\left|\widetilde{v}^{+}\right|^{2 p+2} \mathrm{~d} x}\right] \\
& +\widehat{\alpha}_{1}+\widehat{\alpha}_{2} \\
> & \frac{p \sigma}{2 p+2} \min \left\{\frac{S_{1}^{p+1}}{\mu_{1}\left(\frac{2 p+2}{p} \widehat{\alpha}_{1}+\sigma\right)^{p}}, \frac{S_{2}^{p+1}}{\mu_{2}\left(\frac{2 p+2}{p} \widehat{\alpha}_{2}+\sigma\right)^{p}}\right\}+\widehat{\alpha}_{1}+\widehat{\alpha}_{2},
\end{aligned}
$$


i.e.

$$
\widetilde{J}_{\varepsilon}(\widetilde{u}, \widetilde{v})>\widehat{\alpha}_{1}+\widehat{\alpha}_{2}+\bar{\delta}
$$

where $\bar{\delta}$ is defined in (2.8). Therefore, by (2.22), we may complete the proof of (2.7). Since (2.7) holds for all $(u, v) \in \mathbf{N}_{\varepsilon}$ satisfying (2.13), then we may conclude that

$$
-\varepsilon^{N} \sigma<\int_{\mathbb{R}^{N}} \beta(x)\left|u^{+}\right|^{q+1}\left|v^{+}\right|^{q+1} \mathrm{~d} x \leq 0,
$$

for all $(u, v) \in \mathbf{N}_{\varepsilon}$ with $\varepsilon^{-N} J_{\varepsilon}(u, v) \leq \widehat{\alpha}_{1}+\widehat{\alpha}_{2}+\bar{\delta}$. Hence, by (2.23) and Lemma 2.1, we may obtain (2.10) for all $(u, v) \in \mathbf{N}_{\varepsilon}$ with $\varepsilon^{-N} J_{\varepsilon}(u, v) \leq \widehat{\alpha}_{1}+\widehat{\alpha}_{2}+\bar{\delta}$, provided $0<\sigma<(2 p+2) \sqrt{\widehat{\alpha}_{1} \widehat{\alpha}_{2}}$. This completes the proof.

To prove Theorems 1.2 and 1.3, we need another lemma as follows:

Lemma 2.3 Let $c_{\varepsilon}$ be as in (1.7). Then $\varepsilon^{-N} c_{\varepsilon} \geq \widehat{\alpha}_{1}+\widehat{\alpha}_{2}$ for $\varepsilon>0$ that is sufficiently small. Furthermore, if $\varepsilon^{-N} c_{\varepsilon}=\widehat{\alpha}_{1}+\widehat{\alpha}_{2}$, then problem $\left(P_{\varepsilon}\right)$ does not have a least energy solution.

Proof. By $q \leq p$ and Lemma 2.1, we have

$$
J_{\varepsilon}(u, v) \geq \frac{p}{2 p+2}\|(u, v)\|_{H}^{2} \geq \varepsilon^{N}\left(\widehat{\alpha}_{1}+\widehat{\alpha}_{2}\right) \text { for all }(u, v) \in \mathbf{N}_{\varepsilon} \text { and } \varepsilon>0 .
$$

Hence, $\varepsilon^{-N} c_{\varepsilon} \geq \widehat{\alpha}_{1}+\widehat{\alpha}_{2}$ for all $\varepsilon>0$. Now we want to prove that problem $\left(P_{\varepsilon}\right)$ does not have a least energy solution if $\varepsilon^{-N} c_{\varepsilon}=\widehat{\alpha}_{1}+\widehat{\alpha}_{2}$. Argue by contradiction that there exists $\left(u_{0}, v_{0}\right) \in \mathbf{N}_{\varepsilon}$ that is a least energy solution of problem $\left(P_{\varepsilon}\right)$ such that

$$
J_{\varepsilon}\left(u_{0}, v_{0}\right)=c_{\varepsilon}=\varepsilon^{N}\left(\widehat{\alpha}_{1}+\widehat{\alpha}_{2}\right) .
$$

Hence by the maximum principle for the fractional Laplacian [41], $u_{0}, v_{0}>0$ in $\mathbb{R}^{N}$. So

$$
\int_{\mathbb{R}^{N}} \beta(x)\left|u_{0}\right|^{q+1}\left|v_{0}\right|^{q+1} \mathrm{~d} x<0
$$

and then by Lemma 2.1 and $q \leq p$, we obtain

$$
J_{\varepsilon}\left(u_{0}, v_{0}\right) \geq \frac{p}{2 p+2}\left\|\left(u_{0}, v_{0}\right)\right\|_{H}^{2}>\varepsilon^{N}\left(\widehat{\alpha}_{1}+\widehat{\alpha}_{2}\right)
$$

which would contradict (2.24). Therefore, we may complete the proof.

Now, we need to introduce a generalized barycenter map. By this we mean a continuous map $\Phi$ : $L^{2}\left(\mathbb{R}^{N}\right) \backslash\{0\} \rightarrow \mathbb{R}^{N}$, which is equivariant with respect to the action of the group of euclidian motions in $\mathbb{R}^{N}$, that is, for every $x \in \mathbb{R}^{N}$, every orthogonal $N \times N$ matrix $A$ and every $u \in L^{2}\left(\mathbb{R}^{N}\right) \backslash\{0\}$, one has

$$
\Phi(\xi * u)=\xi+\Phi(u) \text { and } \Phi\left(u \circ A^{-1}\right)=A \Phi(u)
$$

and

$$
\Phi(u \circ \varepsilon)=\varepsilon^{-1} \Phi(u) \text { for all } u \in L^{2}\left(\mathbb{R}^{N}\right) \backslash\{0\} \text { and } \varepsilon>0,
$$

where $(\xi * u)(x)=u(x-\xi)$ and $(u \circ \varepsilon)(x)=u(\varepsilon x)$. This property is easily built into the construction. Indeed, if $\Phi_{1}$ satisfies (2.25) , then $\Phi$ defined by $|u|_{2}^{\frac{2}{N}} \Phi_{1}\left(u \circ|u|_{2}^{\frac{2}{N}}\right)$ satisfies (2.25) and (2.26). Note that the map

$$
u \mapsto \frac{\int_{\mathbb{R}^{N}} x u^{2} \mathrm{~d} x}{\int_{\mathbb{R}^{N}} u^{2} \mathrm{~d} x}
$$

has the invariance properties (2.25) and (2.26), but it is neither well defined on $L^{2}\left(\mathbb{R}^{N}\right) \backslash\{0\}$ nor on $H^{\alpha}\left(\mathbb{R}^{N}\right) \backslash\{0\}$. 
The conditions $\left(D_{1}\right)$ and $\left(D_{2}\right)$ may imply $z_{1, i}^{\varepsilon} \rightarrow P_{1, i}$ and $z_{2, j}^{\varepsilon} \rightarrow P_{2, j}$ as $\varepsilon \rightarrow 0$ (up to a subsequence), where $P_{1, i}$ and $P_{2, j}$ are global minimum points of $V_{1}(x)$ and $V_{2}(x)$, respectively. Generically, $\left(P_{1, i}, P_{2, j}\right)$ may not be equal to $\left(z_{1, i}, z_{2, j}\right)$, because $V_{1}(x)$ and $V_{2}(x)$ may have multiple minimum points. To find the positive solutions $\left(\widehat{u}_{\varepsilon, i}, \widehat{v}_{\varepsilon, j}\right)$ concentrating at $\left(z_{1, i}, z_{2, j}\right)$, we may consider the minimization problem of $J_{\varepsilon}$ over the subset $N_{i, j}(\varepsilon)$ of $\mathbf{N}_{\varepsilon}$, where $N_{i, j_{2}}(\varepsilon)$ is defined by

$$
N_{i, j}(\varepsilon)=\left\{(u, v) \in \mathbf{N}_{\varepsilon}: \Phi(u) \in C_{s}\left(z_{1, i}\right) \text { and } \Phi(v) \in C_{s}\left(z_{2, j}\right)\right\},
$$

where $C_{s}(x)$ is a cube defined by $C_{s}(x)=\prod_{n=1}^{N}\left(x_{n}-s, x_{n}+s\right)$ with the boundary $\partial C_{s}(x)$ for $0<s<$ $r_{0}\left(r_{0}\right.$ is given in $\left(D_{2}\right)$ and $\left.\left(D_{4}\right)\right)$, and $x=\left(x_{1}, \ldots, x_{N}\right) \in \mathbb{R}^{N}$ such that $\overline{C_{s}\left(z_{1, i}\right)} \subset B_{r_{0}}\left(z_{1, i}\right), \overline{C_{s}\left(z_{2, j}\right)} \subset$ $B_{r_{0}}\left(z_{2, j}\right)$ and

$$
\begin{aligned}
& V_{1}(x)>V_{1}\left(z_{1, i}\right) \text { for all } x \in \partial C_{s}\left(z_{1, i}\right) \text { and } i=1,2, \ldots, k, \\
& V_{2}(x)>V_{2}\left(z_{2, j}\right) \text { for all } x \in \partial C_{s}\left(z_{2, j}\right) \text { and } j=1,2, \ldots, \ell .
\end{aligned}
$$

Moreover, by condition $\left(D_{4}\right)$,

$$
\beta(x) \leq-c_{0} \text { for all } x \in \overline{C_{s}\left(z_{1, i}\right)} \text { for all } 1 \leq i \leq m .
$$

Next, we consider the boundary of $N_{i, j}(\varepsilon)$ as follows:

$$
O_{i, j}(\varepsilon)=\left\{(u, v) \in \mathbf{N}_{\varepsilon}: \Phi(u) \in \partial C_{s}\left(z_{1, i}\right) \text { or } \Phi(v) \in \partial C_{s}\left(z_{2, j}\right)\right\} .
$$

Now we consider the minimization of the functional $J_{\varepsilon}$ over $N_{i, j}(\varepsilon)$ and $O_{i, j}(\varepsilon)$, respectively, and denote the corresponding minima as

$$
\gamma_{i, j}(\varepsilon)=\inf _{(u, v) \in N_{i, j}(\varepsilon)} J_{\varepsilon}(u, v) \text { and } \widetilde{\gamma}_{i, j}(\varepsilon)=\inf _{(u, v) \in O_{i, j}(\varepsilon)} J_{\varepsilon}(u, v) .
$$

The upper bound of $\gamma_{i, j}(\varepsilon)$ is given by:

Lemma 2.4 For each $\delta>0$, there exists $\varepsilon_{\delta}>0$ such that for $\varepsilon \in\left(0, \varepsilon_{\delta}\right)$, there holds

$$
\varepsilon^{-N} \gamma_{i, j}(\varepsilon)<\widehat{\alpha}_{1}+\widehat{\alpha}_{2}+\delta \text { for all } 1 \leq i \leq k \text { and } 1 \leq j \leq \ell .
$$

Proof. First, we define test functions $u_{\varepsilon, i}$ and $v_{\varepsilon, j}$ by

$$
\begin{aligned}
& \left(u_{\varepsilon, i}(x), v_{\varepsilon, j}(x)\right) \\
= & \left(\widehat{\omega}_{1}\left(\frac{x-z_{1, i}+x^{\varepsilon}}{\varepsilon}\right) \psi_{\varepsilon}\left(\frac{x-z_{1, i}+x^{\varepsilon}}{\varepsilon}\right), \widehat{\omega}_{2}\left(\frac{x-z_{2, j}-x^{\varepsilon}}{\varepsilon}\right) \psi_{\varepsilon}\left(\frac{x-z_{2, j}-x^{\varepsilon}}{\varepsilon}\right)\right),
\end{aligned}
$$

where $x^{\varepsilon}=\frac{\sqrt{\varepsilon}}{2} e, e \in S^{N-1}=\left\{x \in \mathbb{R}^{N}:|x|=1\right\}$ and $\widehat{\omega}_{l}$ is the unique positive radial solution of Eq. $\left(\widehat{E}_{l}\right)$ for $l=1,2$. Notice that $\widehat{I}_{l}\left(\omega_{l}\right)=\widehat{\alpha}_{l}$ for $l=1,2$. Moreover, for $0<\varepsilon<1$, the function $\psi_{\varepsilon} \in C^{1}\left(\mathbb{R}^{N},[0,1]\right)$ with compact support satisfies

$$
\psi_{\varepsilon}(x)= \begin{cases}1, & |x|<\frac{1}{3 \sqrt{\varepsilon}}-1, \\ 0, & |x|>\frac{1}{3 \sqrt{\varepsilon}}\end{cases}
$$

and $\left|\nabla \psi_{\varepsilon}\right| \leq 2$ in $\mathbb{R}^{N}$. Obviously,

$$
\int_{\mathbb{R}^{N}} \beta(x)\left|u_{\varepsilon, i}^{+}\right|^{q+1}\left|v_{\varepsilon, j}^{+}\right|^{q+1} \mathrm{~d} x=0 \quad \text { for } \varepsilon \text { sufficiently small. }
$$


Then by (2.29), (2.30) and Lemma 3.2 of [32], it is easy to find two positive numbers $t_{\varepsilon, i}, s_{\varepsilon, j}$ such that $\left(t_{\varepsilon, i} u_{\varepsilon, i}, s_{\varepsilon, j} v_{\varepsilon, j}\right) \in \mathbf{N}_{\varepsilon}$ when $\varepsilon$ is sufficiently small and $\left(t_{\varepsilon, i}, s_{\varepsilon, j}\right) \rightarrow(1,1)$ as $\varepsilon \rightarrow 0^{+}$, uniformly for $e \in S^{N-1}$. Moreover, by (2.25), (2.26) and $\left(t_{\varepsilon, i}, s_{\varepsilon, j}\right) \rightarrow(1,1)$, as $\varepsilon \rightarrow 0^{+}$, we have

$$
\begin{aligned}
\Phi\left(t_{\varepsilon, i} u_{\varepsilon, i}\right) & =z_{1, i}-x^{\varepsilon}+\varepsilon \Phi\left(\widehat{\omega}_{1} \psi_{\varepsilon}\right)+o_{\varepsilon}(1) \\
& =z_{1, i}+o_{\varepsilon}(1) .
\end{aligned}
$$

Similarly, $\Phi\left(s_{\varepsilon, j} v_{\varepsilon, j}\right)=z_{2, j}+o_{\varepsilon}(1)$. Thus, $\Phi\left(t_{\varepsilon, i} u_{\varepsilon, i}\right) \in C_{s}\left(z_{1, i}\right)$ and $\Phi\left(s_{\varepsilon, j} v_{\varepsilon, j}\right) \in C_{s}\left(z_{2, j}\right)$ for $\varepsilon$ sufficiently small, and $\left(t_{\varepsilon, i} u_{\varepsilon, i}, s_{\varepsilon, j} v_{\varepsilon, j}\right) \in N_{i, j}(\varepsilon)$. On the other hand, by (2.29), (2.30) and $\left(t_{\varepsilon, i}, s_{\varepsilon, j}\right) \rightarrow$ $(1,1)$ as $\varepsilon \rightarrow 0^{+}$, it is easy to check that

$$
\varepsilon^{-N} J_{\varepsilon}\left(t_{\varepsilon, i} u_{\varepsilon, i}, s_{\varepsilon, j} v_{\varepsilon, j}\right) \rightarrow \widehat{\alpha}_{1}+\widehat{\alpha}_{2} \text { as } \varepsilon \rightarrow 0^{+} \text {uniformly in } e \in S^{N-1} .
$$

Here we have used the facts that $V_{1}\left(z_{1, i}\right)=\lambda_{1}$ and $V_{2}\left(z_{2, j}\right)=\lambda_{2}$. Therefore, by (2.28) and (2.31), we may complete the proof of Lemma 2.4.

On the other hand, we may describe the lower bound of $\widetilde{\gamma}(\varepsilon)$ as follows:

Lemma 2.5 There exist positive numbers $\widetilde{\delta}$ and $\varepsilon_{\widetilde{\delta}}$ such that for every $\varepsilon \in\left(0, \varepsilon_{\widetilde{\delta}}\right)$

$$
\varepsilon^{-N} \widetilde{\gamma}_{i, j}(\varepsilon)>\widehat{\alpha}_{1}+\widehat{\alpha}_{2}+\widetilde{\delta} \text { for all } 1 \leq i \leq k \text { and } 1 \leq j \leq \ell
$$

Proof. We shall prove Lemma 2.5 by contradiction. Suppose there exist $1 \leq i \leq k, 1 \leq j \leq \ell$ and a sequence $\left\{\varepsilon_{n}\right\}_{n=1}^{\infty} \subset \mathbb{R}^{+}$such that $\varepsilon_{n} \rightarrow 0$ and

$$
\varepsilon_{n}^{-N} \widetilde{\gamma}_{i, j}\left(\varepsilon_{n}\right) \rightarrow \widehat{\alpha}_{1}+\widehat{\alpha}_{2}
$$

as $n \rightarrow \infty$. Then there exists $\left\{\left(u_{n}, v_{n}\right)\right\}_{n=1}^{\infty}$ such that $\left(u_{n}, v_{n}\right) \in O_{i, j}\left(\varepsilon_{n}\right)$ for $n \in \mathbb{N}$, and

$$
\varepsilon_{n}^{-N} J_{\varepsilon_{n}}\left(u_{n}, v_{n}\right) \rightarrow \widehat{\alpha}_{1}+\widehat{\alpha}_{2} \text { as } n \rightarrow \infty .
$$

Let $\widetilde{u}_{n}(x)=u_{n}\left(\varepsilon_{n} x\right)$ and $\widetilde{v}_{n}(x)=v_{n}\left(\varepsilon_{n} x\right)$. From (2.32), there holds

$$
\widetilde{J}_{\varepsilon_{n}}\left(\widetilde{u}_{n}, \widetilde{v}_{n}\right) \rightarrow \widehat{\alpha}_{1}+\widehat{\alpha}_{2} \text { as } n \rightarrow \infty .
$$

It follows from (2.33) and Lemma 2.2 that

$$
\int_{\mathbb{R}^{N}} \beta\left(\varepsilon_{n} x\right)\left|\widetilde{u}_{n}^{+}\right|^{q+1}\left|\widetilde{v}_{n}^{+}\right|^{q+1} \mathrm{~d} x \rightarrow 0 \text { as } n \rightarrow \infty .
$$

Since $\left(u_{n}, v_{n}\right) \in O_{i, j}\left(\varepsilon_{n}\right)$ for $n \in \mathbb{N}$, then from (2.34), we obtain

$$
\int_{\mathbb{R}^{N}}\left(\left|(-\triangle)^{\frac{\alpha}{2}} \widetilde{u}_{n}\right|^{2}+V_{1}\left(\varepsilon_{n} x\right) \widetilde{u}_{n}^{2}\right) \mathrm{d} x=\mu_{1} \int_{\mathbb{R}^{N}}\left|\widetilde{u}_{n}^{+}\right|^{2 p+2} \mathrm{~d} x+o(1)
$$

and

$$
\int_{\mathbb{R}^{N}}\left(\left|(-\triangle)^{\frac{\alpha}{2}} \widetilde{v}_{n}\right|^{2}+V_{2}\left(\varepsilon_{n} x\right) \widetilde{v}_{n}^{2}\right) \mathrm{d} x=\mu_{2} \int_{\mathbb{R}^{N}}\left|\widetilde{v}_{n}^{+}\right|^{2 p+2} \mathrm{~d} x+o(1) .
$$

Therefore, by (2.35), (2.36) and a similar argument to the proof of Lemma 3.3 in [36] (or see [7, lemma $3.1]$ ), we may identify a contradiction. This completes the proof. 


\section{Palais-Smale Sequences}

Fix $0<\sigma<(2 p+2) \sqrt{\widehat{\alpha}_{1} \widehat{\alpha}_{2}}$ arbitrarily. Then by Lemma 2.2 ,

$$
-\sigma \varepsilon^{N}<\int_{\mathbb{R}^{N}} \beta(x)\left|u^{+}\right|^{q+1}\left|v^{+}\right|^{q+1} \mathrm{~d} x \leq 0
$$

and

$$
\begin{aligned}
& {\left[p^{2} \mu_{1} \mu_{2} \int_{\mathbb{R}^{N}}\left|u^{+}\right|^{2 p+2} \mathrm{~d} x \int_{\mathbb{R}^{N}}\left|v^{+}\right|^{2 p+2} \mathrm{~d} x-\left(\int_{\mathbb{R}^{N}} \beta(x)\left|u^{+}\right|^{q+1}\left|v^{+}\right|^{q+1} \mathrm{~d} x\right)^{2}\right] } \\
> & \varepsilon^{2 N}\left(4(p+1)^{2} \widehat{\alpha}_{1} \widehat{\alpha}_{2}-\sigma^{2}\right)>0,
\end{aligned}
$$

for all $(u, v) \in N_{i, j}(\varepsilon)$ with $\varepsilon^{-N} J_{\varepsilon}(u, v) \leq \widehat{\alpha}_{1}+\widehat{\alpha}_{2}+\delta_{0}$, where $\delta_{0}=\delta_{0}(\sigma)>0$. Moreover, by Lemmas 2.4 and 2.5, there exist $\delta_{0}>0$ and $\varepsilon_{0}=\varepsilon_{0}\left(\delta_{0}\right)>0$ such that

$$
\gamma(\varepsilon)<\varepsilon^{N}\left(\widehat{\alpha}_{1}+\widehat{\alpha}_{2}+\delta_{0}\right)<\widetilde{\gamma}_{i, j}(\varepsilon) \text { for all } \varepsilon \in\left(0, \varepsilon_{0}\right) .
$$

Here, we may set $0<\delta_{0} \leq \min \left\{\bar{\delta}, \widetilde{\delta}, \widehat{\alpha}_{1}, \widehat{\alpha}_{2}\right\} / 2$, then we have the following results.

Lemma 3.1 For each $(u, v) \in N_{i, j}(\varepsilon)$ with

$$
J_{\varepsilon}(u, v)<\min \left\{\varepsilon^{N}\left(\widehat{\alpha}_{1}+\widehat{\alpha}_{2}+\delta_{0}\right), \widetilde{\gamma}_{i, j}(\varepsilon)\right\},
$$

there exist $b>0$ and a differentiable function

$$
(s, t): B(0 ; b) \equiv\left\{(\widetilde{u}, \widetilde{v}) \in H:\|(\widetilde{u}, \widetilde{v})\|_{H}<b\right\} \rightarrow \mathbb{R}^{+} \times \mathbb{R}^{+}
$$

such that $(s(0,0), t(0,0))=(1,1)$, the function $(s(\widetilde{u}, \widetilde{v})(u-\widetilde{u}), t(\widetilde{u}, \widetilde{v})(v-\widetilde{v})) \in N_{i, j}(\varepsilon)$ and

$$
J_{\varepsilon}(s(\widetilde{u}, \widetilde{v})(u-\widetilde{u}), t(\widetilde{u}, \widetilde{v})(v-\widetilde{v}))<\min \left\{\varepsilon^{N}\left(\widehat{\alpha}_{1}+\widehat{\alpha}_{2}+\delta_{0}\right), \widetilde{\gamma}_{i, j}(\varepsilon)\right\}
$$

for all $(\widetilde{u}, \widetilde{v}) \in B(0 ; b)$. Moreover,

$$
\left(\begin{array}{c}
\left\langle s^{\prime}(0,0),(\phi, \psi)\right\rangle \\
\left\langle t^{\prime}(0,0),(\phi, \psi)\right\rangle
\end{array}\right)=\frac{\left(\begin{array}{c}
\Psi_{1}(u, v, \phi, \psi) K_{2}(u, v)-(q+1) \Psi_{2}(u, v, \phi, \psi) L_{\beta}(u, v) \\
-(q+1) \Psi_{1}(u, v, \phi, \psi) L_{\beta}(u, v)+\Psi_{2}(u, v, \phi, \psi) K_{1}(u, v)
\end{array}\right)}{\Gamma(u, v)}
$$

where

$$
\begin{gathered}
L_{\beta}(u, v)=\int_{\mathbb{R}^{N}} \beta(x)\left|u^{+}\right|^{q+1}\left|v^{+}\right|^{q+1} \mathrm{~d} x, \\
K_{1}(u, v)=2 p \mu_{1} \int_{\mathbb{R}^{N}}\left|u^{+}\right|^{2 p+2} \mathrm{~d} x+(q-1) L_{\beta}(u, v), \\
K_{2}(u, v)=2 p \mu_{2} \int_{\mathbb{R}^{N}}\left|v^{+}\right|^{2 p+2} \mathrm{~d} x+(q-1) L_{\beta}(u, v), \\
\Psi_{1}(u, v, \phi, \psi)=2 \int_{\mathbb{R}^{N}}\left(\varepsilon^{2 \alpha}(-\triangle)^{\frac{\alpha}{2}} u(-\triangle)^{\frac{\alpha}{2}} \phi+V_{1}(x) u \phi\right) \mathrm{d} x \\
-(q+1) \int_{\mathbb{R}^{N}} \beta(x)\left(\left|u^{+}\right|^{q-1} u^{+}\left|v^{+}\right|^{q+1} \phi+\left|u^{+}\right|^{q+1}\left|v^{+}\right|^{q-1} v^{+} \psi\right) \mathrm{d} x \\
-(2 p+2) \int_{\mathbb{R}^{N}} \mu_{1}\left|u^{+}\right|^{2 p} u^{+} \phi \mathrm{d} x, \forall(\phi, \psi) \in H,
\end{gathered}
$$




$$
\begin{aligned}
\Psi_{2}(u, v, \phi, \psi)= & 2 \int_{\mathbb{R}^{N}}\left(\varepsilon^{2 \alpha}(-\triangle)^{\frac{\alpha}{2}} v(-\triangle)^{\frac{\alpha}{2}} \psi+V_{2}(x) v \psi\right) \mathrm{d} x \\
& -(q+1) \int_{\mathbb{R}^{N}} \beta(x)\left(\left|u^{+}\right|^{q-1} u^{+}\left|v^{+}\right|^{q+1} \phi+\left|u^{+}\right|^{q+1}\left|v^{+}\right|^{q-1} v^{+} \psi\right) \mathrm{d} x \\
& -(2 p+2) \int_{\mathbb{R}^{N}} \mu_{2}\left|v^{+}\right|^{2 p} v^{+} \psi \mathrm{d} x, \forall(\phi, \psi) \in H,
\end{aligned}
$$

and

$$
\begin{aligned}
\Gamma(u, v)= & 4 p^{2} \mu_{1} \mu_{2} \int_{\mathbb{R}^{N}}\left|u^{+}\right|^{2 p+2} \mathrm{~d} x \int_{\mathbb{R}^{N}}\left|v^{+}\right|^{2 p+2} \mathrm{~d} x-4 q\left[L_{\beta}(u, v)\right]^{2} \\
& +2 p(q-1) L_{\beta}(u, v)\left[\mu_{1} \int_{\mathbb{R}^{N}}\left|u^{+}\right|^{2 p+2} \mathrm{~d} x+\mu_{2} \int_{\mathbb{R}^{N}}\left|v^{+}\right|^{2 p+2} \mathrm{~d} x\right] .
\end{aligned}
$$

Proof. Let $L_{\beta}(u, v)=\int_{\mathbb{R}^{N}} \beta(x)\left|u^{+}\right|^{q+1}\left|v^{+}\right|^{q+1} \mathrm{~d} x$. Define a function $F: H \times \mathbb{R}^{2} \rightarrow \mathbb{R}^{2}$ given by

$$
F_{(u, v)}\left(w_{1}, w_{2}, s, t\right)=\left(\begin{array}{c}
F_{1}\left(w_{1}, w_{2}, s, t\right) \\
F_{2}\left(w_{1}, w_{2}, s, t\right)
\end{array}\right),
$$

where

$$
\begin{aligned}
& F_{1}\left(w_{1}, w_{2}, s, t\right)=s\left\|u-w_{1}\right\|_{V_{1}}^{2}-\mu_{1} s^{2 p+1} \int_{\mathbb{R}^{N}}\left|\left(u-w_{1}\right)^{+}\right|^{2 p+2} \mathrm{~d} x \\
& -s^{q} t^{q+1} L_{\beta}\left(u-w_{1}, v-w_{2}\right)
\end{aligned}
$$

and

$$
\begin{aligned}
F_{2}\left(w_{1}, w_{2}, s, t\right)= & t\left\|v-w_{2}\right\|_{V_{2}}^{2}-\mu_{2} t^{2 p+1} \int_{\mathbb{R}^{N}}\left|\left(v-w_{2}\right)^{+}\right|^{2 p+2} \mathrm{~d} x \\
& -s^{q+1} t^{q} L_{\beta}\left(u-w_{1}, v-w_{2}\right),
\end{aligned}
$$

Because $(u, v) \in N_{i, j}(\varepsilon)$ and $J_{\varepsilon}(u, v)<\min \left\{\varepsilon^{N}\left(\widehat{\alpha}_{1}+\widehat{\alpha}_{2}+\delta_{0}\right), \widetilde{\gamma}_{i, j}(\varepsilon)\right\}$, we have $F(0,0,1,1)=(0,0)$ and $\|(u, v)\|_{H} \leq C$ for some $C>0$. By a direct computation, we obtain

$$
\begin{gathered}
\frac{\partial}{\partial s} F_{1}(0,0,1,1)=-2 p \mu_{1} \int_{\mathbb{R}^{N}}\left|u^{+}\right|^{2 p+2} \mathrm{~d} x-(q-1) L_{\beta}(u, v), \\
\frac{\partial}{\partial t} F_{1}(0,0,1,1)=\frac{\partial}{\partial s} F_{2}(0,0,1,1)=-(q+1) L_{\beta}(u, v),
\end{gathered}
$$

and

$$
\frac{\partial}{\partial t} F_{2}(0,0,1,1)=-2 p \mu_{2} \int_{\mathbb{R}^{N}}\left|v^{+}\right|^{2 p+2} \mathrm{~d} x-(q-1) L_{\beta}(u, v) .
$$

Hence, by (2.10), we obtain

$$
\begin{aligned}
\left|\begin{array}{ll}
\frac{\partial}{\partial s} F_{1}(0,0,1,1) & \frac{\partial}{\partial t} F_{1}(0,0,1,1) \\
\frac{\partial}{\partial s} F_{2}(0,0,1,1) & \frac{\partial}{\partial t} F_{2}(0,0,1,1)
\end{array}\right|= & {\left[2 p \mu_{1} \int_{\mathbb{R}^{N}}\left|u^{+}\right|^{2 p+2} \mathrm{~d} x+(q-1) L_{\beta}(u, v)\right] } \\
& \times\left[2 p \mu_{2} \int_{\mathbb{R}^{N}}\left|v^{+}\right|^{2 p+2} \mathrm{~d} x+(q-1) L_{\beta}(u, v)\right] \\
& -(q+1)^{2}\left[L_{\beta}(u, v)\right]^{2} \\
> & 0,
\end{aligned}
$$

since $\beta(x) \leq 0$ in $\mathbb{R}^{N}, 0<q \leq p \leq 1$ if $N \leq 4 \alpha$ and $0<q \leq p \leq \frac{2 \alpha}{N-2 \alpha}$ if $N>4 \alpha$. Thus, in view of the Implicit Function Theorem, there exists a differentiable function

$$
(s, t): B(0 ; b) \subset H\left(\mathbb{R}^{N}\right) \rightarrow \mathbb{R}^{+} \times \mathbb{R}^{+}
$$


such that $(s(0), t(0))=(1,1)$ and

$$
F_{(u, v)}\left(w_{1}, w_{2}, s(\widetilde{u}, \widetilde{v}), t(\widetilde{u}, \widetilde{v})\right)=(0,0) \text { for all }(\widetilde{u}, \widetilde{v}) \in B(0 ; b)
$$

This function is equivalent to $(s(\widetilde{u}, \widetilde{v})(u-\widetilde{u}), t(\widetilde{u}, \widetilde{v})(v-\widetilde{v})) \in \mathbf{N}_{\varepsilon}$. Moreover, by the continuity of $J_{\varepsilon}$ and $(s, t)$, we have

$$
(s(\widetilde{u}, \widetilde{v})(u-\widetilde{u}), t(\widetilde{u}, \widetilde{v})(v-\widetilde{v})) \in N_{i, j}(\varepsilon)
$$

and

$$
J_{\varepsilon}(s(\widetilde{u}, \widetilde{v})(u-\widetilde{u}), t(\widetilde{u}, \widetilde{v})(v-\widetilde{v}))<\min \left\{\varepsilon^{N}\left(\widehat{\alpha}_{1}+\widehat{\alpha}_{2}+\delta_{0}\right), \widetilde{\gamma}_{i, j}(\varepsilon)\right\},
$$

if $b$ is sufficiently small.

Now we may use Lemma 3.1 to find a $(P S)_{\gamma_{i, j}(\varepsilon)}$ sequence as follows:

Proposition 3.2 For each $1 \leq i \leq k, 1 \leq j \leq \ell$ and $\varepsilon \in\left(0, \varepsilon_{0}\right)$, there exists a sequence $\left\{\left(u_{n}, v_{n}\right)\right\} \subset$ $N_{i, j}(\varepsilon)$ such that

$$
J_{\varepsilon}\left(u_{n}, v_{n}\right)=\gamma_{i, j}(\varepsilon)+o(1) \text { and } J_{\varepsilon}^{\prime}\left(u_{n}, v_{n}\right)=o(1) \text { in } H^{*} \text { as } n \rightarrow \infty .
$$

Proof. By the Ekeland variational principle [15], there exists a minimizing sequence $\left\{\left(u_{n}, v_{n}\right)\right\} \subset$ $N_{i, j}(\varepsilon)$ such that

$$
J_{\varepsilon}\left(u_{n}, v_{n}\right)<\gamma_{i, j}(\varepsilon)+\frac{1}{n}<\min \left\{\varepsilon^{N}\left(\widehat{\alpha}_{1}+\widehat{\alpha}_{2}+\delta_{0}\right), \widetilde{\gamma}_{i, j}(\varepsilon)\right\}
$$

and

$$
J_{\varepsilon}\left(u_{n}, v_{n}\right)<J_{\varepsilon}\left(w_{1}, w_{2}\right)+\frac{1}{n}\left\|\left(w_{1}, w_{2}\right)-\left(u_{n}, v_{n}\right)\right\|_{H}
$$

for $\left(w_{1}, w_{2}\right) \in \mathbf{N}_{\varepsilon}$ and $n \geq n_{0}$, where $n_{0}$ is a sufficiently large constant independent of $\left(w_{1}, w_{2}\right)$. Applying Lemma 3.1 for each $\left(u_{n}, v_{n}\right)$, we obtain the function

$$
\left(s_{n}, t_{n}\right): B\left(0 ; b_{n}\right) \rightarrow \mathbb{R}^{+} \times \mathbb{R}^{+}
$$

such that $\left(s_{n}(\widetilde{u}, \widetilde{v})\left(u_{n}-\widetilde{u}\right), t_{n}(\widetilde{u}, \widetilde{v})\left(v_{n}-\widetilde{v}\right)\right) \in N_{i, j}(\varepsilon)$, for $(\widetilde{u}, \widetilde{v}) \in B\left(0 ; b_{n}\right)$, where $b_{n}>0$. Fix $(u, v) \in H \backslash\{(0,0)\}$ arbitrarily. For $0<\rho<b_{n}$, let $\left(\phi_{\rho}, \psi_{\rho}\right)=(\rho u, \rho v)\|(u, v)\|_{H}^{-1}, y_{\rho}=s_{n, \rho} u_{n, \rho}$ and $z_{\rho}=t_{n, \rho} v_{n, \rho}$. Hereafter, $s_{n, \rho}=s_{n}\left(\phi_{\rho}, \psi_{\rho}\right), t_{n, \rho}=t_{n}\left(\phi_{\rho}, \psi_{\rho}\right), u_{n, \rho}=u_{n}-\phi_{\rho}$ and $v_{n, \rho}=v_{n}-\psi_{\rho}$. Then $\left(y_{\rho}, z_{\rho}\right) \in N_{i, j}(\varepsilon)$. By (3.4), there holds

$$
J_{\varepsilon}\left(y_{\rho}, z_{\rho}\right)-J_{\varepsilon}\left(u_{n}, v_{n}\right) \geq-\frac{1}{n}\left\|\left(y_{\rho}, z_{\rho}\right)-\left(u_{n}, v_{n}\right)\right\|_{H} .
$$

Because $\left(y_{\rho}, z_{\rho}\right) \rightarrow\left(u_{n}, v_{n}\right)$ as $\rho \rightarrow 0^{+}$, it follows from (3.5) that

$$
\begin{aligned}
& -\left\langle J_{\varepsilon}^{\prime}\left(u_{n}, v_{n}\right),\left(\phi_{\rho}, \psi_{\rho}\right)\right\rangle+\left\langle J_{\varepsilon}^{\prime}\left(u_{n}, v_{n}\right),\left(\left(s_{n, \rho}-1\right) u_{n, \rho},\left(t_{n, \rho}-1\right) v_{n, \rho}\right)\right\rangle \\
\geq & -\frac{1}{n}\left\|\left(y_{\rho}, z_{\rho}\right)-\left(u_{n}, v_{n}\right)\right\|_{H}+o\left(\left\|\left(y_{\rho}, z_{\rho}\right)-\left(u_{n}, v_{n}\right)\right\|_{H}\right) .
\end{aligned}
$$

On the other hand, due to $\left(y_{\rho}, z_{\rho}\right)=\left(s_{n, \rho} u_{n, \rho}, t_{n, \rho} v_{n, \rho}\right) \in N_{i, j}(\varepsilon)$, we have

$$
\begin{aligned}
\int_{\mathbb{R}^{N}}\left(\varepsilon^{2 \alpha}\left|(-\triangle)^{\frac{\alpha}{2}} u_{n, \rho}\right|^{2}+V_{1}(x) u_{n, \rho}^{2}\right) \mathrm{d} x= & \mu_{1} s_{n, \rho}^{2 p} \int_{\mathbb{R}^{N}}\left|u_{n, \rho}^{+}\right|^{2 p+2} \mathrm{~d} x \\
& +s_{n, \rho}^{q-1} t_{n, \rho}^{q+1} \int_{\mathbb{R}^{N}} \beta(x)\left|u_{n, \rho}^{+}\right|^{q+1}\left|v_{n, \rho}^{+}\right|^{q+1} \mathrm{~d} x
\end{aligned}
$$


and

$$
\begin{aligned}
\int_{\mathbb{R}^{N}}\left(\varepsilon^{2 \alpha}\left|(-\triangle)^{\frac{\alpha}{2}} v_{n, \rho}\right|^{2}+V_{2}(x) v_{n, \rho}^{2}\right) \mathrm{d} x= & \mu_{2} t_{n, \rho}^{2 p} \int_{\mathbb{R}^{N}}\left|u_{n, \rho}^{+}\right|^{2 p+2} \mathrm{~d} x \\
& +t_{n, \rho}^{q-1} s_{n, \rho}^{q+1} \int_{\mathbb{R}^{N}} \beta(x)\left|u_{n, \rho}^{+}\right|^{q+1}\left|v_{n, \rho}^{+}\right|^{q+1} \mathrm{~d} x .
\end{aligned}
$$

Then it is easy to verify that

$$
\begin{aligned}
& \left\langle J_{\varepsilon}^{\prime}\left(y_{\rho}, z_{\rho}\right),\left(\left(s_{n, \rho}-1\right) u_{n, \rho},\left(t_{n, \rho}-1\right) v_{n, \rho}\right)\right\rangle \\
= & \left\langle J_{\varepsilon}^{\prime}\left(s_{n, \rho} u_{n, \rho}, t_{n, \rho} v_{n, \rho}\right),\left(\left(s_{n, \rho}-1\right) u_{n, \rho},\left(t_{n, \rho}-1\right) v_{n, \rho}\right)\right\rangle \\
= & 0 .
\end{aligned}
$$

Combining (3.6) and (3.7), we obtain

$$
\begin{aligned}
& \left\langle J_{\varepsilon}^{\prime}\left(u_{n}, v_{n}\right),(u, v)\|(u, v)\|_{H}^{-1}\right\rangle \\
\leq & \frac{\left\|\left(y_{\rho}, z_{\rho}\right)-\left(u_{n}, v_{n}\right)\right\|_{H}}{n \rho}+\frac{o\left(\left\|\left(y_{\rho}, z_{\rho}\right)-\left(u_{n}, v_{n}\right)\right\|_{H}\right)}{\rho} \\
& +\left\langle J_{\varepsilon}^{\prime}\left(u_{n}, v_{n}\right)-J_{\varepsilon}^{\prime}\left(y_{\rho}, z_{\rho}\right),\left(\frac{s_{n, \rho}-1}{\rho} u_{n, \rho}, \frac{t_{n, \rho}-1}{\rho} v_{n, \rho}\right)\right\rangle .
\end{aligned}
$$

By (3.2) and (3.3), there exists a positive constant $C$ that is independent of $\rho$ and $n$ such that

$$
\lim _{\rho \rightarrow 0^{+}} \frac{\left|s_{n, \rho}-1\right|}{\rho} \leq C, \quad \lim _{\rho \rightarrow 0^{+}} \frac{\left|t_{n, \rho}-1\right|}{\rho} \leq C,
$$

and

$$
\left\|\left(y_{\rho}, z_{\rho}\right)-\left(u_{n}, v_{n}\right)\right\|_{H} \leq C\left(\rho+\max \left\{\left|s_{n, \rho}-1\right|,\left|t_{n, \rho}-1\right|\right\}\right) .
$$

Setting $\rho \rightarrow 0^{+}$on (3.8), we obtain

$$
\left\langle J_{\varepsilon}^{\prime}\left(u_{n}, v_{n}\right),(u, v)\|(u, v)\|_{H}^{-1}\right\rangle \leq \frac{C}{n} .
$$

Here we have used (3.9), (3.10) and the fact that $\left(y_{\rho}, z_{\rho}\right) \rightarrow\left(u_{n}, v_{n}\right)$ as $\rho \rightarrow 0^{+}$. Therefore,

$$
\left\|J_{\varepsilon}^{\prime}\left(u_{n}, v_{n}\right)\right\|_{H^{*}} \leq \frac{C}{n} \text { for } n \geq n_{0} .
$$

Therefore, by (3.11), we may complete the proof of Proposition 3.2.

To prove Theorem 1.2, we need the (PS) condition of $J_{\varepsilon}$ as follows:

Proposition 3.3 Assume that $\left\{\left(u_{n}, v_{n}\right)\right\}$ is a sequence in $N_{i, j}(\varepsilon)$ satisfying

(i) $J_{\varepsilon}\left(u_{n}, v_{n}\right) \rightarrow \varepsilon^{N} \theta_{\varepsilon}$ as $n \rightarrow \infty$, where $\theta_{\varepsilon}<\widehat{\alpha}_{1}+\widehat{\alpha}_{2}+\delta_{0}$;

(ii) $J_{\varepsilon}^{\prime}\left(u_{n}, v_{n}\right) \rightarrow 0$ strongly in $H^{*}$ as $n \rightarrow \infty$.

Then there exists a convergent subsequence such that as $n \rightarrow \infty,\left(u_{n}, v_{n}\right) \rightarrow\left(u_{0}, v_{0}\right)$ strongly in $H$, where $\left(u_{0}, v_{0}\right) \in N_{i, j}(\varepsilon)$.

Proof. It follows from (1.6) and $(i)$ that

$$
\frac{p}{2 p+2}\left\|\left(u_{n}, v_{n}\right)\right\|_{H}^{2} \leq J_{\varepsilon}\left(u_{n}, v_{n}\right) \rightarrow \varepsilon^{N} \theta_{\varepsilon} \text { as } n \rightarrow \infty
$$

and $\left\{\left(u_{n}, v_{n}\right)\right\}$ is bounded in $H$. Thus, there exists a convergent subsequence of $\left\{\left(u_{n}, v_{n}\right)\right\}$ (denoted as $\left\{\left(u_{n}, v_{n}\right)\right\}$ for notation convenience) such that as $n \rightarrow \infty$,

$$
\left(u_{n}, v_{n}\right) \rightarrow\left(u_{0}, v_{0}\right) \text { weakly in } H,
$$


where $\left(u_{0}, v_{0}\right) \in H$. By $0<q \leq p \leq 1$ if $N \leq 4 \alpha, 0<q \leq p<\frac{2 \alpha}{N-2 \alpha}$ if $N>4 \alpha$, then in view of (3.12) and the Sobolev compact embedding, we have

$$
\left(u_{n}, v_{n}\right) \rightarrow\left(u_{0}, v_{0}\right) \text { strongly in } L_{l o c}^{r}\left(\mathbb{R}^{N}\right) \times L_{l o c}^{r}\left(\mathbb{R}^{N}\right) \text { for } 2<r<2_{\alpha}^{*}
$$

and

$$
\left(u_{n}, v_{n}\right) \rightarrow\left(u_{0}, v_{0}\right) \text { a.e. in } \mathbb{R}^{N} .
$$

Now we claim that there exist a subsequence $\left\{\left(u_{n}, v_{n}\right)\right\}$ and a sequence $\left\{z_{n}\right\} \subset \mathbb{R}^{N}$ such that

$$
\int_{B^{N}\left(z_{n}, R\right)}\left|u_{n}^{+}\right|^{q+1}\left|v_{n}^{+}\right|^{q+1} \mathrm{~d} x \geq d_{0}>0, \quad \forall n \in \mathbb{N},
$$

where $d_{0}$ and $R$ are positive constants that are independent of $n$. Suppose on the contrary. Thus, for all $R>0$,

$$
\sup _{x \in \mathbb{R}^{N}} \int_{B^{N}(x, R)}\left|u_{n}^{+}\right|^{q+1}\left|v_{n}^{+}\right|^{q+1} \mathrm{~d} x \rightarrow 0 \text { as } n \rightarrow \infty .
$$

Then, similarly to the argument of Lemma I.1 in [34] (see also [43]), we have

$$
\int_{\mathbb{R}^{N}}\left|u_{n}^{+}\right|^{q+1}\left|v_{n}^{+}\right|^{q+1} \mathrm{~d} x \rightarrow 0 \text { as } n \rightarrow \infty
$$

this implies that

$$
\left.\left|\int_{\mathbb{R}^{N}} \beta(x)\right| u_{n}^{+}\right|^{q+1}\left|v_{n}^{+}\right|^{q+1} \mathrm{~d} x \mid \rightarrow 0 \text { as } n \rightarrow \infty .
$$

Therefore, by $(i i),(3.12),(3.13)$ and (3.16) $)$, the functions $u_{0}$ and $v_{0}$ satisfy

$$
\varepsilon^{2 \alpha}(-\triangle)^{\alpha} u_{0}+V_{1}(x) u_{0}=\mu_{1}\left|u_{0}\right|^{2 p} u_{0}^{+}, \text {in } \mathbb{R}^{N}
$$

and

$$
\varepsilon^{2 \alpha}(-\triangle)^{\alpha} v_{0}+V_{2}(x) u_{0}=\mu_{2}\left|v_{0}\right|^{2 p} v_{0}^{+}, \text {in } \mathbb{R}^{N} .
$$

On the other hand, by hypethesis (ii) and (3.14), we have

$$
u_{0}, v_{0} \geq 0 \text { in } \mathbb{R}^{N} .
$$

Furthermore, it follows from (3.14) and (3.16) that

$$
u_{0} v_{0} \equiv 0 .
$$

Thus, by (3.17)-(3.19) and the strong maximum principle for the fractional Laplacian [41, we have $u_{0} \equiv 0$ or $v_{0} \equiv 0$. Without loss of generality, we may assume that $u_{0} \equiv 0$. By Lemma 2.1 and the concentration-compactness principle (cf. [34, 35]), there are positive constants $R, b$ and a sequence $\left\{x_{n}\right\}_{n=1}^{\infty} \subset \mathbb{R}^{N}$ such that

$$
\int_{B^{N}(0 ; R)}\left|\left(-x_{n} * u_{n}\right)^{+}\right|^{2 p+2} \mathrm{~d} x \geq b \text { for } n \text { sufficiently large. }
$$

By (3.13), (3.20) and $u_{0} \equiv 0$, it is easy to verify that $\left\{x_{n}\right\}$ is an unbounded sequence in $\mathbb{R}^{N}$. Let $\widehat{u}_{n}(x)=\left(-x_{n} * u_{n}\right)(x)$. Then sequence $\left\{\widehat{u}_{n}\right\}$ is bounded in $H^{\alpha}\left(\mathbb{R}^{N}\right)$, so we may assume that there exists $\widehat{u}_{0} \in H^{\alpha}\left(\mathbb{R}^{N}\right)$ such that

$$
\widehat{u}_{n} \rightarrow \widehat{u}_{0} \text { weakly in } H^{\alpha}\left(\mathbb{R}^{N}\right)
$$

and

$$
\widehat{u}_{n} \rightarrow \widehat{u}_{0} \text { strongly in } L_{l o c}^{2 p+2}\left(\mathbb{R}^{N}\right) .
$$


It follows from (3.20) and (3.22) that $\widehat{u}_{0} \not \equiv 0$ in $\mathbb{R}^{N}$. Set $w_{n}=\widehat{u}_{n}-\widehat{u}_{0}$. Then we may divide the proof into the following cases:

Case $I: \int_{\mathbb{R}^{N}}\left(\left|(-\triangle)^{\frac{\alpha}{2}} w_{n}\right|^{2}+\lambda_{1} w_{n}^{2}\right) \mathrm{d} x \rightarrow 0$ as $n \rightarrow \infty$;

Case $I I: \int_{\mathbb{R}^{N}}\left(\left|(-\triangle)^{\frac{\alpha}{2}} w_{n}\right|^{2}+\lambda_{1} w_{n}^{2}\right) \mathrm{d} x \geq b$ for large $n$ and for some constant $b>0$.

Suppose Case $I$ holds. Then

$$
x_{n}=\Phi\left(u_{n}\right)-\Phi\left(\widehat{u}_{0}\right)+o(1),
$$

which implies $\left|\Phi\left(u_{n}\right)\right| \rightarrow \infty$ as $n \rightarrow \infty$, this contradicts $\Phi\left(u_{n}\right) \in C_{s}\left(z_{1, i}\right)$. Here, we have used the fact that $\left\{x_{n}\right\}$ is a unbounded sequence in $\mathbb{R}^{N}$.

Suppose Case $I I$ holds. Since $J_{\varepsilon}^{\prime}\left(u_{n}, v_{n}\right) \rightarrow 0$ strongly in $H^{*}$, then by (3.16), we have

$$
\int_{\mathbb{R}^{N}}\left(\varepsilon^{2 \alpha}\left|(-\triangle)^{\frac{\alpha}{2}} u_{n}\right|^{2}+V_{1}(x) u_{n}^{2}\right) \mathrm{d} x=\mu_{1} \int_{\mathbb{R}^{N}}\left|u_{n}^{+}\right|^{2 p+2} \mathrm{~d} x+o(1)
$$

and

$$
\int_{\mathbb{R}^{N}}\left(\varepsilon^{2 \alpha}\left|(-\triangle)^{\frac{\alpha}{2}} v_{n}\right|^{2}+V_{2}(x) v_{n}^{2}\right) \mathrm{d} x=\mu_{2} \int_{\mathbb{R}^{N}}\left|v_{n}^{+}\right|^{2 p+2} \mathrm{~d} x+o(1) .
$$

By (3.23) and Brézis-Lieb lemma [5], we obtain

$$
\int_{\mathbb{R}^{N}}\left(\varepsilon^{2 \alpha}\left|(-\triangle)^{\frac{\alpha}{2}} w_{n}\right|^{2}+V_{1}(x) w_{n}^{2}\right) \mathrm{d} x=\mu_{1} \int_{\mathbb{R}^{N}}\left|w_{n}^{+}\right|^{2 p+2} \mathrm{~d} x+o(1) .
$$

Because $\int_{\mathbb{R}^{N}}\left(\left|(-\triangle)^{\frac{\alpha}{2}} \omega_{n}\right|^{2}+\lambda_{1} w_{n}^{2}\right) d x \geq b$ for large $n$, it is easy to find a sequence $\left\{s_{n}\right\} \subset \mathbb{R}^{+}$with $s_{n} \rightarrow 1$ as $n \rightarrow \infty$ such that

$$
s_{n}^{2} \int_{\mathbb{R}^{N}}\left(\varepsilon^{2 \alpha}\left|(-\triangle)^{\frac{\alpha}{2}} w_{n}\right|^{2}+\left(-x_{n} * V_{1}\right) w_{n}^{2}\right) \mathrm{d} x=\mu_{1} s_{n}^{2 p+2} \int_{\mathbb{R}^{N}}\left|w_{n}^{+}\right|^{2 p+2} \mathrm{~d} x
$$

and so

$$
\frac{1}{2} \int_{\mathbb{R}^{N}}\left(\varepsilon^{2 \alpha}\left|(-\triangle)^{\frac{\alpha}{2}} w_{n}\right|^{2}+\left(-x_{n} * V_{1}\right) w_{n}^{2}\right) \mathrm{d} x-\frac{\mu_{1}}{2 p+2} \int_{\mathbb{R}^{N}}\left|w_{n}^{+}\right|^{2 p+2} \mathrm{~d} x \geq \varepsilon^{N} \widehat{\alpha}_{1}+o(1) .
$$

Analogously,

$$
\frac{1}{2} \int_{\mathbb{R}^{N}}\left(\varepsilon^{2 \alpha}\left|(-\triangle)^{\frac{\alpha}{2}} v_{n}\right|^{2}+\left(-x_{n} * V_{2}\right) v_{n}^{2}\right) \mathrm{d} x-\frac{\mu_{2}}{2 p+2} \int_{\mathbb{R}^{N}}\left|v_{n}^{+}\right|^{2 p+2} \mathrm{~d} x \geq \varepsilon^{N} \widehat{\alpha}_{2}+o(1) .
$$

Furthermore, $\left\langle J_{\varepsilon}^{\prime}\left(u_{n}, v_{n}\right),\left(x_{n} * \widehat{u}_{0}, 0\right)\right\rangle \rightarrow 0$. By (3.21) and (3.22), we have

$$
\int_{\mathbb{R}^{N}}\left(\varepsilon^{2 \alpha}\left|(-\triangle)^{\frac{\alpha}{2}} \widehat{u}_{0}\right|^{2}+\left(-x_{n} * V_{1}\right) \widehat{u}_{0}^{2}\right) \mathrm{d} x-\mu_{1} \int_{\mathbb{R}^{N}}\left|\widehat{u}_{0}^{+}\right|^{2 p+2} \mathrm{~d} x=o(1),
$$

which implies that

$$
\frac{1}{2} \int_{\mathbb{R}^{N}}\left(\varepsilon^{2 \alpha}\left|(-\triangle)^{\frac{\alpha}{2}} \widehat{u}_{0}\right|^{2}+\left(-x_{n} * V_{1}\right) \widehat{u}_{0}^{2}\right) \mathrm{d} x-\frac{\mu_{1}}{2 p+2} \int_{\mathbb{R}^{N}}\left|\widehat{u}_{0}^{+}\right|^{2 p+2} \mathrm{~d} x \geq \varepsilon^{N} \widehat{\alpha}_{1}+o(1) .
$$

It follows from (1.6), (3.16) and Brézis-Lieb lemma [5] that

$$
\begin{aligned}
J_{\varepsilon}\left(u_{n}, v_{n}\right)= & \frac{1}{2} \int_{\mathbb{R}^{N}}\left(\varepsilon^{2 \alpha}\left|(-\triangle)^{\frac{\alpha}{2}} \widehat{w}_{n}\right|^{2}+\left(-x_{n} * V_{1}\right) \widehat{w}_{n}^{2}\right) \mathrm{d} x-\frac{\mu_{1}}{2 p+2} \int_{\mathbb{R}^{N}}\left|\widehat{w}_{n}^{+}\right|^{2 p+2} \mathrm{~d} x \\
& +\frac{1}{2} \int_{\mathbb{R}^{N}}\left(\varepsilon^{2 \alpha}\left|(-\triangle)^{\frac{\alpha}{2}} \widehat{u}_{0}\right|^{2}+\left(-x_{n} * V_{1}\right) \widehat{u}_{0}^{2}\right) \mathrm{d} x-\frac{\mu_{1}}{2 p+2} \int_{\mathbb{R}^{N}}\left|\widehat{u}_{0}^{+}\right|^{2 p+2} \mathrm{~d} x \\
& +\frac{1}{2} \int_{\mathbb{R}^{N}}\left(\varepsilon^{2 \alpha}\left|(-\triangle)^{\frac{\alpha}{2}} \widehat{v}_{n}\right|^{2}+V_{2}(x) v_{n}^{2}\right) \mathrm{d} x-\frac{\mu_{2}}{2 p+2} \int_{\mathbb{R}^{N}}\left|v_{n}^{+}\right|^{2 p+2} \mathrm{~d} x+o(1) \\
\geq & 2 \varepsilon^{N} \widehat{\alpha}_{1}+\varepsilon^{N} \widehat{\alpha}_{2}+o(1),
\end{aligned}
$$


which implies that

$$
\varepsilon^{N} \theta_{\varepsilon}=\lim _{n \rightarrow \infty} J_{\varepsilon}\left(u_{n}, v_{n}\right) \geq 2 \varepsilon^{N} \widehat{\alpha}_{1}+\varepsilon^{N} \widehat{\alpha}_{2} .
$$

However, $\theta_{\varepsilon}<\widehat{\alpha}_{1}+\widehat{\alpha}_{2}+\delta_{0}$ and $0<\delta_{0} \leq \frac{1}{2} \min \left\{\widehat{\alpha}_{1}, \widehat{\alpha}_{2}\right\}$. Hence, we find a contradiction and complete the proof of (3.15). Let $\left(\widetilde{u}_{n}, \widetilde{v}_{n}\right)=\left(-z_{n} * u_{n},-z_{n} * v_{n}\right)$ for $n \in \mathbb{N}$, where the operation $*$ is defined in (2.25). Then as for (3.12)-(3.13), we have

$$
\begin{aligned}
& \left(\widetilde{u}_{n}, \widetilde{v}_{n}\right) \rightarrow\left(\widetilde{u}_{0}, \widetilde{v}_{0}\right) \text { weakly in } H, \\
& \left(\widetilde{u}_{n}, \widetilde{v}_{n}\right) \rightarrow\left(\widetilde{u}_{0}, \widetilde{v}_{0}\right) \text { strongly in } L_{l o c}^{r}\left(\mathbb{R}^{N}\right) \times L_{l o c}^{r}\left(\mathbb{R}^{N}\right) \text { and for all } 2<r<2_{\alpha}^{*}, \\
& \left(\widetilde{u}_{n}, \widetilde{v}_{n}\right) \rightarrow\left(\widetilde{u}_{0}, \widetilde{v}_{0}\right) \text { a.e. in } \mathbb{R}^{N},
\end{aligned}
$$

where $\left(\widetilde{u}_{0}, \widetilde{v}_{0}\right) \in H$. Besides, it follows from (3.15) and (3.25) that

$$
\int_{B^{N}(0, R)}\left|\widetilde{u}_{0}^{+}\right|^{q+1}\left|\widetilde{v}_{0}^{+}\right|^{q+1} \mathrm{~d} x \geq d_{0}
$$

which implies $\widetilde{u}_{0}^{+} \not \equiv 0$ and $\widetilde{v}_{0}^{+} \not \equiv 0$ in $\mathbb{R}^{N}$. Because $\beta(x)$ and $V_{l}(x)$ are uniformly continuous for each $l \in\{1,2\}$, the sequences $\left\{-z_{n} * \beta\right\}$ and $\left\{-z_{n} * V_{l}\right\}$ are equicontinuous, the Arzelà-Ascoli theorem allows us to assume that $-z_{n} * \beta \rightarrow \beta_{\infty} \in C\left(\mathbb{R}^{N}\right)$ and $-z_{n} * V_{l} \rightarrow V_{l}^{0} \in C\left(\mathbb{R}^{N}\right)$ uniformly on bounded subsets of $\mathbb{R}^{N}$. Then

$$
\begin{aligned}
\int_{\mathbb{R}^{N}}\left(-z_{n} * \beta\right)\left|\widetilde{u}_{n}^{+}\right|^{q-1} \widetilde{u}_{n}^{+}\left|\widetilde{v}_{n}^{+}\right|^{q+1} \varphi_{1} \mathrm{~d} x & \rightarrow \int_{\mathbb{R}^{N}} \beta_{\infty}(x)\left|\widetilde{u}_{0}^{+}\right|^{q-1} \widetilde{u}_{0}^{+}\left|\widetilde{v}_{0}^{+}\right|^{q+1} \varphi_{1} \mathrm{~d} x \text { as } n \rightarrow \infty \\
\int_{\mathbb{R}^{N}}\left(-z_{n} * \beta\right)\left|\widetilde{u}_{n}^{+}\right|^{q+1}\left|\widetilde{v}_{n}^{+}\right|^{q-1} \widetilde{v}_{n}^{+} \varphi_{2} \mathrm{~d} x & \rightarrow \int_{\mathbb{R}^{N}} \beta_{\infty}(x)\left|\widetilde{u}_{0}^{+}\right|^{q+1}\left|\widetilde{v}_{0}^{+}\right|^{q+1} \widetilde{v}_{0}^{+} \varphi_{2} \mathrm{~d} x \text { as } n \rightarrow \infty
\end{aligned}
$$

and

$$
\begin{aligned}
& \int_{\mathbb{R}^{N}}\left(-z_{n} * V_{1}\right) \widetilde{u}_{n} \varphi \mathrm{d} x \rightarrow \int_{\mathbb{R}^{N}} V_{1}^{0}(x) \widetilde{u}_{0} \varphi_{1} \mathrm{~d} x \text { as } n \rightarrow \infty, \\
& \int_{\mathbb{R}^{N}}\left(-z_{n} * V_{2}\right) \widetilde{v}_{n} \varphi_{2} \mathrm{~d} x \rightarrow \int_{\mathbb{R}^{N}} V_{2}^{0}(x) \widetilde{v}_{0} \varphi_{2} \mathrm{~d} x \text { as } n \rightarrow \infty,
\end{aligned}
$$

for all $\varphi_{1}, \varphi_{2} \in C_{0}^{\infty}\left(\mathbb{R}^{N}\right)$. Therefore, by (3.24), (3.25) and the hypothesis $(i i)$, we obtain

$$
\begin{aligned}
& \int_{\mathbb{R}^{N}}\left(\varepsilon^{2 \alpha}(-\triangle)^{\frac{\alpha}{2}} \widetilde{u}_{0}(-\triangle)^{\frac{\alpha}{2}} \varphi_{1}+V_{1}^{0} \widetilde{u}_{0} \varphi_{1}\right) \mathrm{d} x-\mu_{1} \int_{\mathbb{R}^{N}}\left|\widetilde{u}_{0}^{+}\right|^{2 p} \widetilde{u}_{0}^{+} \varphi_{1} \mathrm{~d} x \\
& +\int_{\mathbb{R}^{N}}\left(\varepsilon^{2 \alpha}(-\triangle)^{\frac{\alpha}{2}} \widetilde{v}_{0}(-\triangle)^{\frac{\alpha}{2}} \varphi_{2}+V_{2}^{0} \widetilde{v}_{0} \varphi_{2}\right) \mathrm{d} x-\mu_{2} \int_{\mathbb{R}^{N}}\left|\widetilde{v}_{0}^{+}\right|^{2 p} \widetilde{v}_{0}^{+} \varphi_{2} \mathrm{~d} x \\
& -\int_{\mathbb{R}^{N}} \beta_{\infty}(x)\left|\widetilde{u}_{0}\right|^{q-1} \widetilde{u}_{0}\left|\widetilde{v}_{0}\right|^{q+1} \varphi_{1} \mathrm{~d} x-\int_{\mathbb{R}^{N}} \beta_{\infty}(x)\left|\widetilde{u}_{0}^{+}\right|^{q+1}\left|\widetilde{v}_{0}^{+}\right|^{q-1} \widetilde{v}_{0}^{+} \varphi_{2} \mathrm{~d} x \\
= & \lim _{n \rightarrow \infty}\left\langle J_{\varepsilon}^{\prime}\left(\widetilde{u}_{n}, \widetilde{v}_{n}\right),\left(\varphi_{1}, \varphi_{2}\right)\right\rangle \\
= & \lim _{n \rightarrow \infty}\left\langle J_{\varepsilon}^{\prime}\left(u_{n}, v_{n}\right),\left(z_{n} * \varphi_{1}, z_{n} * \varphi_{2}\right)\right\rangle=0, \quad \forall \varphi_{1}, \varphi_{2} \in C_{0}^{\infty}\left(\mathbb{R}^{N}\right),
\end{aligned}
$$

i.e., $\left(\widetilde{u}_{0}, \widetilde{v}_{0}\right)$ is a nonnegative solution of the following problem

$$
\begin{cases}\varepsilon^{2 \alpha}(-\triangle)^{\alpha} u+V_{1}^{0}(x) u=\left(\mu_{1}|u|^{2 p}+\beta_{\infty}(x)|u|^{q-1}|v|^{q+1}\right) u, & \text { in } \mathbb{R}^{N} \\ \varepsilon^{2 \alpha}(-\triangle)^{\alpha} v+V_{2}^{0}(x) v=\left(\mu_{2}|v|^{2 p}+\beta_{\infty}(x)|v|^{q-1}\left|u^{+}\right|^{q+1}\right) v, & \text { in } \mathbb{R}^{N}\end{cases}
$$

Hence

$$
\int_{\mathbb{R}^{N}}\left(\varepsilon^{2 \alpha}\left|(-\triangle)^{\frac{\alpha}{2}} \widetilde{u}_{0}\right|^{2}+V_{1}^{0}(x) \widetilde{u}_{0}^{2}\right) \mathrm{d} x=\int_{\mathbb{R}^{N}}\left(\mu_{1}\left|\widetilde{u}_{0}^{+}\right|^{2 p+2}+\beta_{\infty}(x)\left|\widetilde{u}_{0}^{+}\right|^{q+1}\left|\widetilde{v}_{0}^{+}\right|^{q+1}\right) \mathrm{d} x
$$


and

$$
\int_{\mathbb{R}^{N}}\left(\varepsilon^{2 \alpha}\left|(-\triangle)^{\frac{\alpha}{2}} \widetilde{v}_{0}\right|^{2}+V_{2}^{0}(x) \widetilde{v}_{0}^{2}\right) \mathrm{d} x=\int_{\mathbb{R}^{N}}\left(\mu_{2}\left|\widetilde{v}_{0}^{+}\right|^{2 p+2}+\beta_{\infty}(x)\left|\widetilde{u}_{0}^{+}\right|^{q+1}\left|\widetilde{v}_{0}^{+}\right|^{q+1}\right) \mathrm{d} x .
$$

Then in view of Lemma 2.3, we obtain

$$
I_{\varepsilon}\left(\widetilde{u}_{0}, \widetilde{v}_{0}\right) \geq \varepsilon^{N}\left(\widehat{\alpha}_{1}+\widehat{\alpha}_{2}\right),
$$

where

$$
\begin{aligned}
I_{\varepsilon}(u, v)= & \frac{1}{2} \int_{\mathbb{R}^{N}}\left(\varepsilon^{2 \alpha}\left|(-\triangle)^{\frac{\alpha}{2}} u\right|^{2}+V_{1}^{0}(x) u^{2}\right) \mathrm{d} x+\frac{1}{2} \int_{\mathbb{R}^{N}}\left(\varepsilon^{2 \alpha}\left|(-\triangle)^{\frac{\alpha}{2}} v\right|^{2}+V_{2}^{0}(x) v^{2}\right) \mathrm{d} x \\
& -\frac{1}{2 p+2}\left(\int_{\mathbb{R}^{N}} \mu_{1}\left|u^{+}\right|^{2 p+2} \mathrm{~d} x+\int_{\mathbb{R}^{N}} \mu_{2}\left|v^{+}\right|^{2 p+2}\right) \mathrm{d} x \\
& -\frac{1}{q+1} \int_{\mathbb{R}^{N}} \beta_{\infty}(x)\left|u^{+}\right|^{q+1}\left|v^{+}\right|^{q+1} \mathrm{~d} x,
\end{aligned}
$$

for $(u, v) \in H$. Let $\left(\widehat{u}_{n}, \widehat{v}_{n}\right)=\left(u_{n}-z_{n} * \widetilde{u}_{0}, v_{n}-z_{n} * \widetilde{v}_{0}\right)$, i.e.

$$
\left(-z_{n} * \widehat{u}_{n},-z_{n} * \widehat{v}_{n}\right)=\left(\widetilde{u}_{n}-\widetilde{u}_{0}, \widetilde{v}_{n}-\widetilde{v}_{0}\right) .
$$

By (3.24), it is evident that

$$
\int_{\mathbb{R}^{N}}\left(\varepsilon^{2 \alpha}\left|(-\triangle)^{\frac{\alpha}{2}}\left(\widetilde{u}_{n}-\widetilde{u}_{0}\right)\right|^{2}-\varepsilon^{2 \alpha}\left|(-\triangle)^{\frac{\alpha}{2}} \widetilde{u}_{n}\right|^{2}+\varepsilon^{2 \alpha}\left|(-\triangle)^{\frac{\alpha}{2}} \widetilde{u}_{0}\right|^{2}\right) \mathrm{d} x=o(1)
$$

and

$$
\int_{\mathbb{R}^{N}}\left(\varepsilon^{2 \alpha}\left|(-\triangle)^{\frac{\alpha}{2}}\left(\widetilde{v}_{n}-\widetilde{v}_{0}\right)\right|^{2}-\varepsilon^{2 \alpha}\left|(-\triangle)^{\frac{\alpha}{2}} \widetilde{v}_{n}\right|^{2}+\varepsilon^{2 \alpha}\left|(-\triangle)^{\frac{\alpha}{2}} \widetilde{v}_{0}\right|^{2}\right) \mathrm{d} x=o(1) .
$$

Furthermore, since $-z_{n} * \beta \rightarrow \beta_{\infty}(x)$ and $-z_{n} * V_{l} \rightarrow V_{l}^{0}(x)$ uniformly on bounded subsets for $l=1,2$, we may conclude that

$$
\int_{\mathbb{R}^{N}}\left[\left(-z_{n} * V_{1}\right)\left(\widetilde{u}_{n}-\widetilde{u}_{0}\right)^{2}-\left(-z_{n} * V_{1}\right) \widetilde{u}_{n}^{2}+V_{1}^{0}(x) \widetilde{u}_{0}^{2}\right] \mathrm{d} x=o(1)
$$

and

$$
\int_{\mathbb{R}^{N}}\left[\left(-z_{n} * V_{2}\right)\left(\widetilde{v}_{n}-\widetilde{v}_{0}\right)^{2}-\left(-z_{n} * V_{2}\right) \widetilde{v}_{n}^{2}+V_{2}^{0}(x) \widetilde{v}_{0}^{2}\right] \mathrm{d} x=o(1) .
$$

On the other hand, it follows from Brézis-Lieb Lemma (cf. [5]) that

$$
\begin{gathered}
\int_{\mathbb{R}^{N}}\left[\left|\left(\widetilde{u}_{n}-\widetilde{u}_{0}\right)^{+}\right|^{2 p+2}-\left|\widetilde{u}_{n}^{+}\right|^{2 p+2}+\left|\widetilde{u}_{0}^{+}\right|^{2 p+2}\right] \mathrm{d} x=o(1), \\
\int_{\mathbb{R}^{N}}\left[\left|\left(\widetilde{v}_{n}-\widetilde{v}_{0}\right)^{+}\right|^{2 p+2}-\left|\widetilde{v}_{n}^{+}\right|^{2 p+2}+\left|\widetilde{v}_{0}^{+}\right|^{2 p+2}\right] \mathrm{d} x=o(1)
\end{gathered}
$$

and

$$
\begin{aligned}
& \int_{\mathbb{R}^{N}}\left(-z_{n} * \beta\right)\left|\left(\widetilde{u}_{n}-\widetilde{u}_{0}\right)^{+}\right|^{q+1}\left|\left(\widetilde{v}_{n}-\widetilde{v}_{0}\right)^{+}\right|^{q+1} \mathrm{~d} x \\
= & \int_{\mathbb{R}^{N}}\left(-z_{n} * \beta\right)\left|\widetilde{u}_{n}^{+}\right|^{q+1}\left|\widetilde{v}_{n}^{+}\right|^{q+1} \mathrm{~d} x+\int_{\mathbb{R}^{N}} \beta_{\infty}(x)\left|\widetilde{u}_{0}^{+}\right|^{q+1}\left|\widetilde{v}_{0}^{+}\right|^{q+1} \mathrm{~d} x+o(1) .
\end{aligned}
$$


By (3.27), (3.28) and (3.30) - (3.36), we have

$$
\begin{aligned}
& \int_{\mathbb{R}^{N}}\left(\varepsilon^{2 \alpha}\left|(-\triangle)^{\frac{\alpha}{2}} \widehat{u}_{n}\right|^{2}+V_{1}(x) \widehat{u}_{n}^{2}\right) \mathrm{d} x=\mu_{1} \int_{\mathbb{R}^{N}}\left|\widehat{u}_{n}^{+}\right|^{2 p+2} \mathrm{~d} x \\
& +\int_{\mathbb{R}^{N}} \beta(x)\left|\widehat{u}_{n}^{+}\right|^{q+1}\left|\widehat{v}_{n}^{+}\right|^{q+1} \mathrm{~d} x+o(1), \\
& \int_{\mathbb{R}^{N}}\left(\varepsilon^{2 \alpha}\left|(-\triangle)^{\frac{\alpha}{2}} \widehat{v}_{n}\right|^{2}+V_{2} \widehat{v}_{n}^{2}\right) \mathrm{d} x=\mu_{2} \int_{\mathbb{R}^{N}}\left|\widehat{v}_{n}^{+}\right|^{2 p+2} \mathrm{~d} x \\
& +\int_{\mathbb{R}^{N}} \beta(x)\left|\widehat{u}_{n}^{+}\right|^{q+1}\left|\widehat{v}_{n}^{+}\right|^{q+1} \mathrm{~d} x+o(1)
\end{aligned}
$$

and

$$
J_{\varepsilon}\left(\widehat{u}_{n}, \widehat{v}_{n}\right)=J_{\varepsilon}\left(u_{n}, v_{n}\right)-I_{\varepsilon}\left(u_{0}, v_{0}\right)+o(1)=\varepsilon^{N} \theta_{\varepsilon}-I_{\varepsilon}\left(u_{0}, v_{0}\right)+o(1) .
$$

Now we want to show the strong convergence of $\left\|\left(\widehat{u}_{n}, \widehat{v}_{n}\right)\right\|_{H} \rightarrow 0$ as $n \rightarrow \infty$. Suppose not, then $\left\|\left(\widehat{u}_{n}, \widehat{v}_{n}\right)\right\|_{H} \geq c_{0}$ for large $n$, where $c_{0}$ is a positive constant independent of $n$. Hence, it follows from (3.37), (3.38), Lemma 2.1 and Lemma 2.3 that

$$
J_{\varepsilon}\left(\widehat{u}_{n}, \widehat{v}_{n}\right) \geq \frac{\varepsilon^{N}}{2} \min \left\{\bar{\delta}, \widehat{\alpha}_{1}, \widehat{\alpha}_{2}\right\}
$$

for $n$ sufficiently large. Thus, in view of (3.29), (3.39) and (3.40), we obtain

$$
\varepsilon^{N} \theta_{\varepsilon} \geq \varepsilon^{N}\left(\widehat{\alpha}_{1}+\widehat{\alpha}_{2}\right)+\frac{\varepsilon^{N}}{2} \min \left\{\bar{\delta}, \widehat{\alpha}_{1}, \widehat{\alpha}_{2}\right\} .
$$

which is a contradiction with $\theta_{\varepsilon}<\widehat{\alpha}_{1}+\widehat{\alpha}_{2}+\delta_{0}$. Hence, the strong convergence $\left\|\left(\widehat{u}_{n}, \widehat{v}_{n}\right)\right\|_{H} \rightarrow 0$ as $n \rightarrow \infty$ holds, i.e.

$$
\left\|\left(u_{n}-z_{n} * \widetilde{u}_{0}, v_{n}-z_{n} * \widetilde{v}_{0}\right)\right\|_{H} \rightarrow 0 \text { as } n \rightarrow \infty .
$$

It follows from (2.25) and (3.41) that

$$
o(1)=\Phi\left(u_{n}\right)-\Phi\left(z_{n} * \widetilde{u}_{0}\right)=\Phi\left(u_{n}\right)-z_{n}-\Phi\left(\widetilde{u}_{0}\right),
$$

i.e.

$$
z_{n}=\Phi\left(u_{n}\right)-\Phi\left(\widetilde{u}_{0}\right)+o(1) .
$$

Since $\Phi\left(u_{n}\right) \in C_{s}\left(z_{1, i}\right)$ for $n \in \mathbb{N}$, then (3.42) implies that $\left\{z_{n}\right\}$ is a bounded sequence in $\mathbb{R}^{N}$. As a result, we may assume $z_{n} \rightarrow z_{0} \in \mathbb{R}^{N}$ as $n \rightarrow \infty$. Thus, (3.41) becomes

$$
\left(u_{n}, v_{n}\right) \rightarrow\left(z_{0} * \widetilde{u}_{0}, z_{0} * \widetilde{v}_{0}\right) \text { strongly in } H .
$$

Since $\left(u_{n}, v_{n}\right) \in N_{i, j}(\varepsilon)$ for $n \in \mathbb{N}$, then by (3.43), we have $\left(z_{0} * \widetilde{u}_{0}, z_{0} * \widetilde{v}_{0}\right) \in N_{i, j}(\varepsilon) \cup O_{i, j}(\varepsilon)$. In view of the assumption $(i)$, (3.1) and (3.43), we obtain

$$
J_{\varepsilon}\left(z_{0} * \widetilde{u}_{0}, z_{0} * \widetilde{v}_{0}\right)=\varepsilon^{N} \theta_{\varepsilon}<\varepsilon^{N}\left(\widehat{\alpha}_{1}+\widehat{\alpha}_{2}+\delta_{0}\right)<\widetilde{\gamma}(\varepsilon),
$$

so $\left(z_{0} * \widetilde{u}_{0}, z_{0} * \widetilde{v}_{0}\right) \notin O_{i, j}(\varepsilon)$, i.e., $\left(z_{0} * \widetilde{u}_{0}, z_{0} * \widetilde{v}_{0}\right) \in N_{i, j}(\varepsilon)$. Therefore, we may complete the proof of Proposition 3.3 .

Theorem 3.4 For each $0<\varepsilon<\varepsilon_{0}, 1 \leq i \leq k$ and $1 \leq j \leq \ell$, problem $\left(P_{\varepsilon}\right)$ has a positive solution $\left(\widehat{u}_{\varepsilon, i}, \widehat{v}_{\varepsilon, j}\right) \in N_{i, j}(\varepsilon)$.

Proof. From Propositions 3.2 and 3.3, we can prove Theorem 3.4. 


\section{Proof of Theorem 1.2}

Throughout this section, we define

$$
\left[\varepsilon^{-N} J_{\varepsilon} \leq d\right]_{i, j}=\left\{(u, v) \in N_{i, j}(\varepsilon): \varepsilon^{-N} J_{\varepsilon}(u, v) \leq d\right\} \text { for } d \in \mathbb{R} .
$$

The main goal of this section is to show

$$
\operatorname{cat}\left(\left[\varepsilon^{-N} J_{\varepsilon} \leq \widehat{\alpha}_{1}+\widehat{\alpha}_{2}+\delta_{\varepsilon}\right]_{i, j}\right) \geq 2 \text { if } z_{1, i}=z_{2, j}
$$

where $0<\delta_{\varepsilon} \rightarrow 0$ as $\varepsilon \rightarrow 0^{+}$. Here $\operatorname{cat}(\cdot)$ is the standard Lusternik-Schnirelman category (cf. [6]).

From Lemma 4.1 in 33. and Lemma 2.2 in [31, we obtain

Lemma 4.1 Let $\left(u_{\varepsilon}, v_{\varepsilon}\right)$ be a constrained critical point of $J_{\varepsilon}$ on $\mathbf{N}_{\varepsilon}$ with

$$
\varepsilon^{-N} J_{\varepsilon}\left(u_{\varepsilon}, v_{\varepsilon}\right) \leq \widehat{\alpha}_{1}+\widehat{\alpha}_{2}+\delta_{0} .
$$

Then $\nabla J_{\varepsilon}\left(u_{\varepsilon}, v_{\varepsilon}\right)=0$ on $H^{*}$.

Therefore, from Theorem 2.3 in [2], Proposition 3.3 and Lemma 4.1, we have

Proposition 4.2 Suppose cat $\left(M_{i, j}\left(\varepsilon, \delta_{0}\right)\right) \geq k$, where $k \in \mathbb{N}$ and

$$
M_{i, j}\left(\varepsilon, \delta_{0}\right)=\left[\varepsilon^{-N} J_{\varepsilon} \leq \widehat{\alpha}_{1}+\widehat{\alpha}_{2}+\delta_{0}\right]_{i, j} .
$$

Then the functional $J_{\varepsilon}$ has at least $k$ critical points in $M_{i, j}\left(\varepsilon, \delta_{0}\right)$.

Now we define a function by

$$
h(u, v)=\Phi(u)-\Phi(v) \text { for }(u, v) \in H .
$$

This leads to the following results.

Lemma 4.3 Suppose that $z_{1, i}=z_{2, j}$. Then there exist $\widetilde{\varepsilon}_{0}>0$ and $0<\delta_{1} \leq \delta_{0}$ such that for every $0<\varepsilon<\widetilde{\varepsilon}_{0}$, there holds

$$
|h(u, v)|>0 \text { for all }(u, v) \in\left[\varepsilon^{-N} J_{\varepsilon} \leq \widehat{\alpha}_{1}+\widehat{\alpha}_{2}+\delta_{1}\right]_{i, j} .
$$

Proof. We may prove this by contradiction. Suppose that there exists $\varepsilon_{n}>0$, for $n \in \mathbb{N}, \varepsilon_{n} \rightarrow 0$ as $n \rightarrow \infty$ and $\left(u_{n}, v_{n}\right) \in N_{i, j}\left(\varepsilon_{n}\right)$ such that

$$
h\left(u_{n}, v_{n}\right)=0, \text { for all } n \in \mathbb{N},
$$

and

$$
\varepsilon_{n}^{-N} J_{\varepsilon_{n}}\left(u_{n}, v_{n}\right)=\widehat{\alpha}_{1}+\widehat{\alpha}_{2}+o(1) .
$$

To obtain a contradiction with (4.2), it is sufficient to show that

$$
\frac{1}{\varepsilon_{n}}\left|h\left(u_{n}, v_{n}\right)\right| \rightarrow \infty \text { as } n \rightarrow \infty .
$$

Then $\Phi\left(u_{n}\right)=\Phi\left(v_{n}\right)$ and

$$
\Phi\left(u_{n}\right), \Phi\left(v_{n}\right) \in C_{s}\left(z_{1, i}\right)=C_{s}\left(z_{2, j}\right)
$$


Let $\widetilde{u}_{n}(x)=u_{n}\left(\varepsilon_{n} x\right)$ and $\widetilde{v}_{n}(x)=v_{n}\left(\varepsilon_{n} x\right)$. Clearly, $\Phi\left(\widetilde{u}_{n}\right)=\Phi\left(\widetilde{v}_{n}\right)$ and $\Phi\left(\widetilde{u}_{n}\right), \Phi\left(\widetilde{v}_{n}\right) \in C_{s / \varepsilon_{n}}\left(z_{1, i}\right)$. By a similar argument to the proof of Lemma 2.5] and the proof of Lemma 3.3 in [36], we can obtain

$$
\int_{\mathbb{R}^{N}}\left(V_{1}\left(\varepsilon_{n} x\right)-\lambda_{1}\right) \widetilde{u}_{n}^{2} \mathrm{~d} x=o(1) \text { and } \int_{\mathbb{R}^{N}}\left(V_{2}\left(\varepsilon_{n} x\right)-\lambda_{2}\right) \widetilde{v}_{n}^{2} \mathrm{~d} x=o(1) .
$$

Moreover, we can conclude that $\widehat{I}_{1}\left(\widetilde{u}_{n}\right)=\widehat{\alpha}_{1}+o(1), \widehat{I}_{2}\left(\widetilde{v}_{n}\right)=\widehat{\alpha}_{2}+o(1)$ and

$$
\begin{aligned}
\int_{\mathbb{R}^{N}}\left(\left|(-\triangle)^{\frac{\alpha}{2}} \widetilde{u}_{n}\right|^{2}+\lambda_{1} \widetilde{u}_{n}^{2}\right) \mathrm{d} x & =\mu_{1} \int_{\mathbb{R}^{N}}\left|\widetilde{u}_{n}^{+}\right|^{2 p+2} \mathrm{~d} x+o(1), \\
\int_{\mathbb{R}^{N}}\left(\left|(-\triangle)^{\frac{\alpha}{2}} \widetilde{v}_{n}\right|^{2}+\lambda_{2} \widetilde{v}_{n}^{2}\right) \mathrm{d} x & =\mu_{2} \int_{\mathbb{R}^{N}}\left|\widetilde{v}_{n}^{+}\right|^{2 p+2} \mathrm{~d} x+o(1) .
\end{aligned}
$$

Thus, there exist $t_{n}, s_{n}>0$ with $t_{n}, s_{n} \rightarrow 1$ as $n \rightarrow \infty$ such that $\bar{u}_{n}=t_{n} \widetilde{u}_{n} \in \widehat{\mathbf{M}}_{1}$ and $\bar{v}_{n}=s_{n} \widetilde{v}_{n} \in \widehat{\mathbf{M}}_{2}$, which implies that $\widehat{I}_{1}\left(\bar{u}_{n}\right)=\widehat{\alpha}_{1}+o(1)$ and $\widehat{I}_{2}\left(\bar{v}_{n}\right)=\widehat{\alpha}_{2}+o(1)$. Furthermore, by the Ekeland varitional principle [15], we may assume that $\left\{\bar{u}_{n}\right\}$ is a $(\mathrm{PS})_{\widehat{\alpha}_{1}}$-sequence of $\widehat{I}_{1}$ in $H^{\alpha}\left(\mathbb{R}^{N}\right)$ and that $\left\{\bar{v}_{n}\right\}$ is a (PS) $\widehat{\alpha}_{2}$-sequence of $\widehat{I}_{2}$ in $H^{\alpha}\left(\mathbb{R}^{N}\right)$, respectively. Applying the concentration-compactness principle of Lions [34, 35], there are positive constants $R, b_{0}$ and two sequences $\left\{x_{n}\right\},\left\{y_{n}\right\} \subset \mathbb{R}^{N}$ such that

$$
\int_{B^{N}\left(x_{n} ; R\right)}\left|\bar{u}_{n}^{+}\right|^{2 p+2} \mathrm{~d} x \geq b_{0} \text { and } \int_{B^{N}\left(y_{n} ; R\right)}\left|\bar{v}_{n}^{+}\right|^{2 p+2} \mathrm{~d} x \geq b_{0} \text { for all } n \in \mathbb{N} .
$$

Let $\widehat{u}_{n}(x)=\bar{u}_{n}\left(x+x_{n}\right)$ for $x \in \mathbb{R}^{N}$ and $n \in \mathbb{N}$. Then, due to translation invariance, it is obvious that $\left\{\widehat{u}_{n}\right\}$ is also a (PS) $\widehat{\alpha}_{1}$-sequence of $\widehat{I}_{1}$ in $H^{\alpha}\left(\mathbb{R}^{N}\right)$. Hence by (4.5), we may assume that there exists a subsequence of $\left\{\widehat{u}_{n}\right\}$ such that

$$
\begin{aligned}
\widehat{u}_{n} & \rightarrow u_{0} \quad \text { in } H^{\alpha}\left(\mathbb{R}^{N}\right), \\
\widehat{u}_{n} & \rightarrow u_{0} \text { a.e. in } \mathbb{R}^{N}, \\
\int_{B^{N}(0 ; R)}\left|\widehat{u}_{n}^{+}\right|^{2 p+2} \mathrm{~d} x & \rightarrow \int_{B^{N}(0 ; R)}\left|u_{0}^{+}\right|^{2 p+2} \mathrm{~d} x \geq b_{0} .
\end{aligned}
$$

Now we set $w_{n}=\widehat{u}_{n}-u_{0}$. Then it follows from (4.6) and Brézis-Lieb Lemma [5] that

$$
\int_{\mathbb{R}^{N}}\left|\widehat{u}_{n}^{+}\right|^{2 p+2} \mathrm{~d} x=\int_{\mathbb{R}^{N}}\left|u_{0}^{+}\right|^{2 p+2} \mathrm{~d} x+\int_{\mathbb{R}^{N}}\left|w_{n}^{+}\right|^{2 p+2} \mathrm{~d} x+o(1)
$$

and

$$
\begin{aligned}
& \int_{\mathbb{R}^{N}}\left(\left|(-\triangle)^{\frac{\alpha}{2}} \widehat{u}_{n}\right|^{2}+\lambda_{1} \widehat{u}_{n}^{2}\right) \mathrm{d} x=\int_{\mathbb{R}^{N}}\left(\left|(-\triangle)^{\frac{\alpha}{2}} u_{0}\right|^{2}+\lambda_{1} u_{0}^{2}\right) \mathrm{d} x \\
& +\int_{\mathbb{R}^{N}}\left(\left|(-\triangle)^{\frac{\alpha}{2}} w_{n}\right|^{2}+\lambda_{1} w_{n}^{2}\right) \mathrm{d} x+o(1) .
\end{aligned}
$$

Combining (4.7), (4.8) and $\left\{\widehat{u}_{n}\right\}$ is a (PS) $\widehat{\alpha}_{1}$-sequence of $\widehat{I}_{1}$ in $H^{\alpha}\left(\mathbb{R}^{N}\right)$, we obtain

$$
\int_{\mathbb{R}^{N}}\left(\left|(-\triangle)^{\frac{\alpha}{2}} w_{n}\right|^{2}+\lambda_{1} w_{n}^{2}\right) \mathrm{d} x=\mu_{1} \int_{\mathbb{R}^{N}}\left|w_{n}^{+}\right|^{2 p+2} \mathrm{~d} x+o(1)
$$

and

$$
\frac{p}{2 p+2} \int_{\mathbb{R}^{N}}\left(\left|(-\triangle)^{\frac{\alpha}{2}} w_{n}\right|^{2}+\lambda_{1} w_{n}^{2}\right) \mathrm{d} x=\widehat{I}_{1}\left(w_{n}\right)+o(1)=\widehat{I}_{1}\left(\widehat{u}_{n}\right)-\widehat{I}_{1}\left(u_{0}\right)+o(1)=o(1) .
$$

Consequently, $\widehat{u}_{n} \rightarrow u_{0}$ strongly in $H^{\alpha}\left(\mathbb{R}^{N}\right)$ and $\widehat{I}_{1}\left(u_{0}\right)=\widehat{\alpha}_{1}$. Similarly, $\widehat{v}_{n} \rightarrow v_{0}$ strongly in $H^{\alpha}\left(\mathbb{R}^{N}\right)$ and $\widehat{I}_{2}\left(v_{0}\right)=\widehat{\alpha}_{2}$, where $\widehat{v}_{n}(x)=\bar{v}_{n}\left(x+y_{n}\right)$ for $x \in \mathbb{R}^{N}$ and $n \in \mathbb{N}$. Therefore, we obtain

$$
\widehat{u}_{n}(x) \rightarrow u_{0}(x) ; \widehat{v}_{n}(x) \rightarrow v_{0}(x) \text { strongly in } H^{\alpha}\left(\mathbb{R}^{N}\right) \text {, }
$$


as $n \rightarrow \infty$, where $u_{0}$ and $v_{0}$ are positive solutions of Eq. $\left(\widehat{E}_{1}\right)$ and Eq. $\left(\widehat{E}_{2}\right)$, respectively (cf. [3] and [20]). From $\Phi\left(\widetilde{u}_{n}\right), \Phi\left(\widetilde{v}_{n}\right) \in C_{s / \varepsilon_{n}}(0) \widehat{u}_{n}(x)=\bar{u}_{n}\left(x+x_{n}\right), \widehat{v}_{n}(x)=\bar{v}_{n}\left(x+y_{n}\right)$ and (4.9), we have

$$
\varepsilon_{n} x_{n}=\varepsilon_{n} \Phi\left(\bar{u}_{n}\right)-\varepsilon_{n} \Phi\left(\widehat{u}_{n}\right)=\varepsilon_{n} \Phi\left(\widetilde{u}_{n}\right)-\varepsilon_{n} \Phi\left(u_{0}\right)
$$

and

$$
\varepsilon_{n} y_{n}=\varepsilon_{n} \Phi\left(\bar{v}_{n}\right)-\varepsilon_{n} \Phi\left(\widehat{v}_{n}\right)=\varepsilon_{n} \Phi\left(\widetilde{v}_{n}\right)-\varepsilon_{n} \Phi\left(v_{0}\right)
$$

and so

$$
\operatorname{dist}\left(\varepsilon_{n} x_{n}, C_{s}\left(z_{1, i}\right)\right) \rightarrow 0 \text { and } \operatorname{dist}\left(\varepsilon_{n} y_{n}, C_{s}\left(z_{2, j}\right)\right) \rightarrow 0 \text { as } n \rightarrow \infty \text {. }
$$

Without loss of generality, we may assume $\varepsilon_{n} x_{n} \rightarrow x_{0} \in \overline{C_{s}\left(z_{1, i}\right)}, \varepsilon_{n} y_{n} \rightarrow y_{0} \in \overline{C_{s}\left(z_{2, j}\right)}$. By condition $\left(D_{2}\right)$, we must have that

$$
V_{1}\left(x_{0}\right)=\lambda_{1}=V_{1}\left(z_{1, i}\right)
$$

and

$$
V_{2}\left(y_{0}\right)=\lambda_{2}=V_{2}\left(z_{2, j}\right),
$$

this implies that $x_{0}=y_{0}=z_{1, i}=z_{2, j}$. Moreover,

$$
\Phi\left(u_{0}\right)=\Phi\left(-x_{n} * \bar{u}_{n}\right)+o(1)=-x_{n}+\Phi\left(\widetilde{u}_{n}\right)+o(1)
$$

and

$$
\Phi\left(v_{0}\right)=\Phi\left(-y_{n} * \bar{v}_{n}\right)+o(1)=-y_{n}+\Phi\left(\widetilde{v}_{n}\right)+o(1)
$$

which implies that

$$
x_{n}-y_{n} \rightarrow z_{0}:=\Phi\left(u_{0}\right)-\Phi\left(v_{0}\right) \text { as } n \rightarrow \infty .
$$

It follows from condition $\left(D_{4}\right), \varepsilon_{n} x_{n} \rightarrow 0$ as $n \rightarrow \infty$, (2.27) and (4.9) that

$$
\begin{aligned}
\int_{\mathbb{R}^{N}} \beta\left(\varepsilon_{n} x\right)\left|\bar{u}_{n}^{+}\right|^{q+1}\left|\bar{v}_{n}^{+}\right|^{q+1} \mathrm{~d} x & =\int_{\mathbb{R}^{N}} \beta\left(\varepsilon_{n} x\right)\left|\widehat{u}_{n}^{+}\left(x-x_{n}\right)\right|^{q+1}\left|\widehat{v}_{n}^{+}\left(x-y_{n}\right)\right|^{q+1} \mathrm{~d} x \\
& =\int_{\mathbb{R}^{N}} \beta\left(\varepsilon_{n} x+\varepsilon_{n} x_{n}\right)\left|\widehat{u}_{n}^{+}(x)\right|^{q+1}\left|\widehat{v}_{n}^{+}\left(x+x_{n}-y_{n}\right)\right|^{q+1} \mathrm{~d} x \\
& \leq \frac{1}{2} \int_{C_{s / \varepsilon_{n}}\left(z_{1, i}\right)} \beta\left(\varepsilon_{n} x+z_{1, i}\right)\left|u_{0}^{+}(x)\right|^{q+1}\left|v_{0}^{+}\left(x+z_{0}\right)\right|^{q+1} \\
& \leq-\frac{c_{0}}{2} \int_{\mathbb{R}^{N}}\left|u_{0}^{+}(x)\right|^{q+1}\left|v_{0}^{+}\left(x+z_{0}\right)\right|^{q+1} \mathrm{~d} x
\end{aligned}
$$

for $n$ sufficiently large. However, by (4.3) and Lemma 2.2, we obtain

$$
\int_{\mathbb{R}^{N}} \beta\left(\varepsilon_{n} x\right)\left|\bar{u}_{n}^{+}\right|^{q+1}\left|\bar{v}_{n}^{+}\right|^{q+1} \mathrm{~d} x \rightarrow 0 \text { as } n \rightarrow \infty,
$$

which is a contradiction with (4.11). This completes the proof.

For $0<\varepsilon<\widetilde{\varepsilon}_{0}$, a map $F_{\varepsilon}^{(i, j)}: S^{N-1} \rightarrow H$ can be written as

$$
F_{\varepsilon}^{(i, j)}(e)=\left(t_{\varepsilon, i} u_{\varepsilon, i}, s_{\varepsilon, j} v_{\varepsilon, j}\right) \text { for } e \in S^{N-1},
$$

where $\widetilde{\varepsilon}_{0}$ is given by Lemma 4.3. Here $\left(t_{\varepsilon, i} u_{\varepsilon, i}, s_{\varepsilon, j} v_{\varepsilon, j}\right)$ is as in the proof of Lemma 2.4. Note that $\left(t_{\varepsilon, i}, s_{\varepsilon, j}\right) \rightarrow(1,1)$ as $\varepsilon \rightarrow 0^{+}$and by (2.31)

$$
\varepsilon^{-N} J_{\varepsilon}\left(t_{\varepsilon, i} u_{\varepsilon, i}, s_{\varepsilon, j} v_{\varepsilon, j}\right) \rightarrow \widehat{\alpha}_{1}+\widehat{\alpha}_{2} \text { as } \varepsilon \rightarrow 0^{+} \text {uniformly in } e \in S^{N-1} .
$$

Then, from (4.12) and Lemma 2.3, we obtain

$$
\varepsilon^{-N} J_{\varepsilon}\left(F_{\varepsilon}^{(i, j)}(e)\right)<\widehat{\alpha}_{1}+\widehat{\alpha}_{2}+\delta_{\varepsilon} \text { uniformly in } e \in S^{N-1},
$$


where

$$
0<\delta_{\varepsilon}=2 \max _{e \in S^{N-1}}\left[\varepsilon^{-N} J_{\varepsilon}\left(F_{\varepsilon}^{(i, j)}(e)\right)-\widehat{\alpha}_{1}-\widehat{\alpha}_{2}\right] \rightarrow 0 \text { as } \varepsilon \rightarrow 0 .
$$

Here we have used the facts that the map $F_{\varepsilon}$ is continuous and that the set $F_{\varepsilon}\left(S^{N-1}\right)$ is compact. As a result, (4.13) and (4.14) imply:

Lemma 4.4 Suppose that $z_{1, i}=z_{2, j}$. Then there exists $0<\sigma_{\varepsilon}<\delta_{\varepsilon}$ such that

$$
F_{\varepsilon}^{(i, j)}\left(S^{N-1}\right) \subset\left[\varepsilon^{-N} J_{\varepsilon} \leq \widehat{\alpha}_{1}+\widehat{\alpha}_{2}+\delta_{\varepsilon}-\sigma_{\varepsilon}\right]_{i, j} .
$$

By (4.14) and Lemma 4.3, we may define

$$
G_{\varepsilon}^{(i, j)}:\left[\varepsilon^{-N} J_{\varepsilon} \leq \widehat{\alpha}_{1}+\widehat{\alpha}_{2}+\delta_{\varepsilon}\right]_{i, j} \rightarrow S^{N-1}
$$

by

$$
G_{\varepsilon}^{(i, j)}(u, v)=-\frac{h(u, v)}{|h(u, v)|} \text { for }(u, v) \in\left[\varepsilon^{-N} J_{\varepsilon} \leq \widehat{\alpha}_{1}+\widehat{\alpha}_{2}+\delta_{\varepsilon}\right]_{i, j} .
$$

Then we can prove the following results.

Lemma 4.5 Suppose that $z_{1, i}=z_{2, j}$. Then there exists a positive number $\varepsilon_{*} \leq \widetilde{\varepsilon}_{0}$ such that for $0<\varepsilon<\varepsilon_{*}$, the $\operatorname{map} G_{\varepsilon}^{(i, j)} \circ F_{\varepsilon}^{(i, j)}: S^{N-1} \rightarrow S^{N-1}$ is homotopic to the identity.

Proof. Let $\bar{G}_{\varepsilon}^{(i, j)}: \Theta \rightarrow S^{N-1}$ satisfy

$$
\bar{G}_{\varepsilon}^{(i, j)}(u, v)=-\frac{h(u, v)}{|h(u, v)|} \text { for }(u, v) \in \Theta
$$

where $\Theta=\{(u, v) \in H \backslash\{0,0\}:|h(u, v)|>0\}$. By Lemma 4.3. one may regard the map $\bar{G}_{\varepsilon}^{(i, j)}$ as an extension of $G_{\varepsilon}^{(i, j)}$. By $\left(t_{\varepsilon, i}, s_{\varepsilon, j}\right) \rightarrow(1,1)$ as $\varepsilon \rightarrow 0^{+}$, for $\theta \in[0,1 / 2)$,

$$
(1-2 \theta) F_{\varepsilon}^{(i, j)}(e)+2 \theta\left(u_{\varepsilon, i}, v_{\varepsilon, j}\right)=\left(u_{\varepsilon, i}, v_{\varepsilon, j}\right)+o_{\varepsilon}(1) \text { in } H^{\alpha}\left(\mathbb{R}^{N}\right) \times H^{\alpha}\left(\mathbb{R}^{N}\right) \text { as } \varepsilon \rightarrow 0^{+} .
$$

Hence as for the proof of Lemma 4.3, there exists a positive number $\varepsilon_{*} \leq \widetilde{\varepsilon}_{0}$ such that for $0<\varepsilon<\varepsilon_{*}$, there holds,

$$
(1-2 \theta) F_{\varepsilon}^{(i, j)}(e)+2 \theta\left(u_{\varepsilon, i}, v_{\varepsilon, j}\right) \in \Theta \text { for all } e \in S^{N-1} \text { and } \theta \in[0,1 / 2) .
$$

On the other hand, for $e \in S^{N-1}$ and $\theta \in[1 / 2,1)$, we may set

$$
f_{\varepsilon, i}(x, \theta)=\omega_{1}\left(\frac{x-z_{1, i}}{\varepsilon}+\frac{e}{4 \sqrt{\varepsilon}(1-\theta)}\right) \psi_{\varepsilon}\left(\frac{x-z_{1, i}}{\varepsilon}+\frac{e}{4 \sqrt{\varepsilon}(1-\theta)}\right)
$$

and

$$
g_{\varepsilon, j}(x, \theta)=\omega_{2}\left(\frac{x-z_{2, j}}{\varepsilon}-\frac{e}{4 \sqrt{\varepsilon}(1-\theta)}\right) \psi_{\varepsilon}\left(\frac{x-z_{2, j}}{\varepsilon}-\frac{e}{4 \sqrt{\varepsilon}(1-\theta)}\right),
$$

where $z_{1, i}$ and $z_{2, j}$ are minimum points of $V_{1}(x)$ and $V_{2}(x)$, respectively. Then it is easy to verify that

$$
\Phi\left(f_{\varepsilon, i}\right)=z_{1, i}-\frac{\sqrt{\varepsilon}}{4(1-\theta)} e+O(\varepsilon) \text { and } \Phi\left(g_{\varepsilon, j}\right)=z_{2, j}+\frac{\sqrt{\varepsilon}}{4(1-\theta)} e+O(\varepsilon),
$$

which implies that

$$
h\left(f_{\varepsilon, i}, g_{\varepsilon, j}\right)=\Phi\left(f_{\varepsilon, i}\right)-\Phi\left(g_{\varepsilon, j}\right)=z_{1, i}-z_{2, j}-\frac{\sqrt{\varepsilon}}{2(1-\theta)} e+O(\varepsilon),
$$


for $e \in S^{N-1}$ and $\theta \in[1 / 2,1)$. Consequently, due to $z_{1, i}=z_{2, j}$,

$$
\left(f_{\varepsilon, i}(\cdot, \theta), g_{\varepsilon, j}(\cdot, \theta)\right) \in \Theta \text { for all } e \in S^{N-1} \text { and } \theta \in[1 / 2,1) .
$$

One may remark that to assure $\left|h\left(f_{\varepsilon, i}, g_{\varepsilon, j}\right)\right|>0$ for $e \in S^{N-1}$ and $\theta \in[1 / 2,1)$, it is necessary for $z_{1, i}$ to be a common minimum point of the $V_{1}(x)$ and $V_{2}(x)$. Otherwise, if $V_{1}(x)$ and $V_{2}(x)$ have two different minimum points at $z_{1, i}$ and $z_{2, j}$, one may use (4.16) to find some $e \sim \frac{z_{1, i}-z_{2, j}}{\left|z_{1, i}-z_{2, j}\right|} \in S^{N-1}$ and $\theta \sim 1-\frac{\sqrt{\varepsilon}}{2\left|z_{1, i}-z_{2, j}\right|} \in[1 / 2,1)$ such that $h\left(f_{\varepsilon, i}, g_{\varepsilon, j}\right)=0$ and $\left(f_{\varepsilon, i}, g_{\varepsilon, j}\right) \notin \Theta$.

By (4.15) and (4.17), we may define

$$
\zeta_{\varepsilon}(\theta, e):[0,1] \times S^{N-1} \rightarrow S^{N-1}
$$

by

$$
\zeta_{\varepsilon}^{(i, j)}(\theta, e)= \begin{cases}\bar{G}_{\varepsilon}^{(i, j)}\left((1-2 \theta) F_{\varepsilon}^{(i, j)}(e)+2 \theta\left(u_{\varepsilon}, v_{\varepsilon}\right)\right), & \text { for } \theta \in[0,1 / 2) \\ \bar{G}_{\varepsilon}^{(i, j)}\left(f_{\varepsilon, i}(\cdot, \theta), g_{\varepsilon, j}(\cdot, \theta)\right), & \text { for } \theta \in[1 / 2,1) \\ e, & \text { for } \theta=1 .\end{cases}
$$

Then $\zeta_{\varepsilon}^{(i, j)}(0, e)=\bar{G}_{\varepsilon}^{(i, j)}\left(F_{\varepsilon}^{(i, j)}(e)\right)=G_{\varepsilon}^{(i, j)}\left(F_{\varepsilon}^{(i, j)}(e)\right)$ and $\zeta_{\varepsilon}^{(i, j)}(1, e)=e$. It is easy to verify that

$$
\lim _{\theta \rightarrow \frac{1}{2}^{-}} \zeta_{\varepsilon}^{(i, j)}(\theta, e)=\bar{G}_{\varepsilon}^{(i, j)}\left(f_{\varepsilon, i}\left(\cdot, \frac{1}{2}\right), g_{\varepsilon, j}\left(\cdot, \frac{1}{2}\right)\right) .
$$

On the other hand, by (4.16) and $z_{1, i}=z_{2, j}$, we have

$$
\lim _{\theta \rightarrow 1^{-}} \zeta_{\varepsilon}^{(i, j)}(\theta, e)=e \text { for } e \in S^{N-1} .
$$

Consequently, by (4.18) and (4.19), we know that $\zeta_{\varepsilon}^{(i, j)} \in C\left([0,1] \times S^{N-1}, S^{N-1}\right)$ and

$$
\begin{array}{cc}
\zeta_{\varepsilon}^{(i, j)}(0, e)=G_{\varepsilon}^{(i, j)}\left(F_{\varepsilon}^{(i, j)}(e)\right) & \text { for all } e \in S^{N-1}, \\
\zeta_{\varepsilon}^{(i, j)}(1, e),=e & \text { for all } e \in S^{N-1},
\end{array}
$$

provided that $0<\varepsilon<\varepsilon_{*}$. Therefore, we may complete the proof.

By Lemma 4.5 and Adachi-Tanaka's Lemma 2.5 (cf. [1]), we obtain

$$
\operatorname{cat}\left(\left[\varepsilon^{-N} J_{\varepsilon} \leq \widehat{\alpha}_{1}+\widehat{\alpha}_{2}+\delta_{\varepsilon}\right]_{i, j}\right) \geq 2,
$$

for $z_{1, i}=z_{2, j}$ and $0<\varepsilon<\varepsilon_{*}$. Thus, by Proposition 4.2, $J_{\varepsilon}$ has at least two critical points in $M_{i, j}\left(\varepsilon, \delta_{\varepsilon}\right)$, where $M_{i, j}\left(\varepsilon, \delta_{\varepsilon}\right)=\left[\varepsilon^{-N} J_{\varepsilon} \leq \widehat{\alpha}_{1}+\widehat{\alpha}_{2}+\delta_{\varepsilon}\right]_{i, j}$. This implies that problem $\left(P_{\varepsilon}\right)$ has two positive solutions $\left(\widehat{u}_{\varepsilon, i}, \widehat{v}_{\varepsilon, j}\right),\left(\widetilde{u}_{\varepsilon, i}, \widetilde{v}_{\varepsilon, j}\right) \in N_{i, j}(\varepsilon)$ such that $\left|h\left(\widehat{u}_{\varepsilon, i}, \widehat{v}_{\varepsilon, j}\right)\right|>0$ and $\left|h\left(\widetilde{u}_{\varepsilon, i}, \widetilde{v}_{\varepsilon, j}\right)\right|>0$. So we may conclude that:

Theorem 4.6 Suppose that $z_{1, i}=z_{2, j}$. Then for each $0<\varepsilon<\varepsilon_{*}$, problem $\left(P_{\varepsilon}\right)$ has at least two positive solutions $\left(\widehat{u}_{\varepsilon, i}, \widehat{v}_{\varepsilon, j}\right),\left(\widetilde{u}_{\varepsilon, i}, \widetilde{v}_{\varepsilon, j}\right) \in M_{i, j}\left(\varepsilon, \delta_{\varepsilon}\right)$ such that

$$
\left|h\left(\widehat{u}_{\varepsilon, i}, \widehat{v}_{\varepsilon, j}\right)\right|>0 \text { and }\left|h\left(\widetilde{u}_{\varepsilon, i}, \widetilde{v}_{\varepsilon, j}\right)\right|>0 .
$$

We are now ready to prove Theorem 1.2, By Theorem 3.4 and Theorem 4.6, we can conclude that problem $\left(P_{\varepsilon}\right)$ has at least $k \times \ell+m$ positive solutions. As for the proofs of (4.9) and (4.10), we may use (4.14) and a similar argument to that in the proof of [32, Theorem 2.4], we can conclude that part (ii) is holds. 


\section{Proof of Theorem 1.3}

By Lemmas 2.2 and 2.4 there exists a positive function $\delta_{\varepsilon}$ with $\delta_{\varepsilon} \rightarrow 0$ as $\varepsilon \rightarrow 0$ such that the sublevel set

$$
\widehat{M}\left(\varepsilon, \delta_{\varepsilon}\right)=\left\{(u, v) \in \mathbf{N}_{\varepsilon}: \varepsilon^{-N} J_{\varepsilon}(u, v) \leq \widehat{\alpha}_{1}+\widehat{\alpha}_{2}+\delta_{\varepsilon}\right\}
$$

is nonempty. Then we have the following results.

Lemma 5.1 Assume that $\liminf _{|x| \rightarrow \infty} V_{l}(x) \equiv V_{l, \infty}>\lambda_{l}$ for all $l=1,2$. Then

$$
\lim _{\varepsilon \rightarrow 0} \sup _{(u, v) \in \widehat{M}\left(\varepsilon, \delta_{\varepsilon}\right)} \inf _{(x, y) \in \mathbf{B}}|(\Phi(u), \Phi(v))-(x, y)|=0 .
$$

where $\mathbf{B}=\underset{1 \leq i \leq k, 1 \leq j \leq \ell}{\cup}\left[C_{s / 2}\left(z_{1, i}\right) \times C_{s / 2}\left(z_{2, j}\right)\right]$.

Proof. Let $\varepsilon_{n} \rightarrow 0$ as $n \rightarrow \infty$; for any $n \in \mathbb{N}$, there exists $\left(u_{n}, v_{n}\right) \in \widehat{M}\left(\varepsilon, \delta_{\varepsilon_{n}}\right)$ such that

$$
\inf _{(x, y) \in \mathbf{B}}\left|\left(\Phi\left(u_{n}\right), \Phi\left(v_{n}\right)\right)-(x, y)\right|=\sup _{(u, v) \in \widehat{M}\left(\varepsilon, \delta_{\varepsilon_{n}}\right)} \inf _{(x, y) \in \mathbf{B}}|(\Phi(u), \Phi(v))-(x, y)|+o(1) .
$$

To prove (5.1), it is sufficient to find points $\left(x_{n}, y_{n}\right) \in \mathbf{B}$ such that

$$
\lim _{n \rightarrow \infty}\left|\left(\Phi\left(u_{n}\right), \Phi\left(v_{n}\right)\right)-\left(x_{n}, y_{n}\right)\right|=0,
$$

possibly up to subsequence. For any $n \in \mathbb{N}$, let $\widetilde{u}_{n}(x)=u_{n}\left(\varepsilon_{n} x\right)$ and $\widetilde{v}_{n}(x)=v_{n}\left(\varepsilon_{n} x\right)$. Then similar to the argument in the proof of Lemma 2.5, we have

$$
\lim _{n \rightarrow \infty} \int_{\mathbb{R}^{N}}\left(\left|(-\triangle)^{\frac{\alpha}{2}} \widetilde{u}_{n}\right|^{2}+V_{1}\left(\varepsilon_{n} x\right) \widetilde{u}_{n}^{2}\right) \mathrm{d} x=\frac{2 p+2}{p} \widehat{\alpha}_{1}
$$

and

$$
\lim _{n \rightarrow \infty} \int_{\mathbb{R}^{N}}\left(\left|(-\triangle)^{\frac{\alpha}{2}} \widetilde{v}_{n}\right|^{2}+V_{2}\left(\varepsilon_{n} x\right) \widetilde{v}_{n}^{2}\right) \mathrm{d} x=\frac{2 p+2}{p} \widehat{\alpha}_{2} .
$$

Furthermore, there exists $\left\{\left(\widetilde{x}_{n}, \widetilde{y}_{n}\right)\right\} \subset \mathbb{R}^{N} \times \mathbb{R}^{N}$ such that

(i) $\widetilde{u}_{n}\left(\cdot+\widetilde{x}_{n}\right)$ converges strongly in $H^{\alpha}\left(\mathbb{R}^{N}\right)$ to $\widehat{u}_{0}$, a positive ground state solution of $\left(\widehat{E}_{1}\right)$;

(ii) $\widetilde{v}_{n}\left(\cdot+\widetilde{y}_{n}\right)$ converges strongly in $H^{\alpha}\left(\mathbb{R}^{N}\right)$ to $\widehat{v}_{0}$, a positive ground state solution of $\left(\widehat{E}_{2}\right)$.

Let us prove that $\left\{\left(\varepsilon_{n} \widetilde{x}_{n}, \varepsilon_{n} \widetilde{y}_{n}\right)\right\}$ is a bounded sequence in $\mathbb{R}^{N} \times \mathbb{R}^{N}$. Arguing by contradiction, we assume that $\left|\varepsilon_{n} \widetilde{x}_{n}\right| \rightarrow \infty$ as $n \rightarrow \infty$. Then

$$
\begin{aligned}
& \lim _{n \rightarrow \infty} \int_{\mathbb{R}^{N}}\left(\left|(-\triangle)^{\frac{\alpha}{2}} \widetilde{u}_{n}\right|^{2}+V_{1}\left(\varepsilon_{n} x\right) \widetilde{u}_{n}^{2}\right) \mathrm{d} x \\
= & \lim _{n \rightarrow \infty} \int_{\mathbb{R}^{N}}\left(\left|(-\triangle)^{\frac{\alpha}{2}} \widetilde{u}_{n}\left(x+\widetilde{x}_{n}\right)\right|^{2}+V_{1}\left(\varepsilon_{n} x+\varepsilon_{n} \widetilde{x}_{n}\right) \widetilde{u}_{n}^{2}\left(x+\widetilde{x}_{n}\right)\right) \mathrm{d} x \\
\geq & \int_{\mathbb{R}^{N}}\left(\left|(-\triangle)^{\frac{\alpha}{2}} u_{0}\right|^{2}+V_{1, \infty} u_{0}^{2}\right) \mathrm{d} x>\int_{\mathbb{R}^{N}}\left(\left|(-\triangle)^{\frac{\alpha}{2}} u_{0}\right|^{2}+\lambda_{1} u_{0}^{2}\right) \mathrm{d} x \\
= & \frac{2 p+2}{p} \widehat{\alpha}_{1},
\end{aligned}
$$


which is a contradiction. Thus, $\left\{\left(\varepsilon_{n} \widetilde{x}_{n}, \varepsilon_{n} \widetilde{y}_{n}\right)\right\}$ is bounded and converges to some $\left(x_{0}, y_{0}\right)$ (up to a subsequence). We are left to prove $\left(x_{0}, y_{0}\right)=\left(z_{1, i}, z_{2, j}\right)$ for some $1 \leq i \leq k, 1 \leq j \leq \ell$. Because

$$
\begin{aligned}
\frac{2 p+2}{p} \widehat{\alpha}_{1} & =\int_{\mathbb{R}^{N}}\left(\left|(-\triangle)^{\frac{\alpha}{2}} u_{0}\right|^{2}+\lambda_{1} u_{0}^{2}\right) \mathrm{d} x \\
& =\lim _{n \rightarrow \infty} \int_{\mathbb{R}^{N}}\left[\left|(-\triangle)^{\frac{\alpha}{2}} \widetilde{u}_{n}\left(x+\widetilde{x}_{n}\right)\right|^{2}+V_{1}\left(\varepsilon_{n} x+\varepsilon_{n} \widetilde{x}_{n}\right) \widetilde{u}_{n}^{2}\left(x+\widetilde{x}_{n}\right)\right] \mathrm{d} x \\
& =\int_{\mathbb{R}^{N}}\left(\left|(-\triangle)^{\frac{\alpha}{2}} u_{0}\right|^{2}+V_{1, \infty}\left(x_{0}\right) u_{0}^{2}\right) \mathrm{d} x
\end{aligned}
$$

and

$$
\begin{aligned}
\frac{2 p+2}{p} \widehat{\alpha}_{2} & =\int_{\mathbb{R}^{N}}\left(\left|(-\triangle)^{\frac{\alpha}{2}} v_{0}\right|^{2}+\lambda_{2} v_{0}^{2}\right) \mathrm{d} x \\
& =\lim _{n \rightarrow \infty} \int_{\mathbb{R}^{N}}\left[\left|(-\triangle)^{\frac{\alpha}{2}} \widetilde{v}_{n}\left(x+\widetilde{y}_{n}\right)\right|^{2}+V_{2}\left(\varepsilon_{n} x+\varepsilon_{n} \widetilde{y}_{n}\right) \widetilde{v}_{n}^{2}\left(x+\widetilde{y}_{n}\right)\right] \mathrm{d} x \\
& =\int_{\mathbb{R}^{N}}\left(\left|(-\triangle)^{\frac{\alpha}{2}} v_{0}\right|^{2}+V_{2, \infty}\left(y_{0}\right) v_{0}^{2}\right) \mathrm{d} x
\end{aligned}
$$

which implies that $V_{1, \infty}\left(x_{0}\right)=\lambda_{1}$ and $V_{2, \infty}\left(y_{0}\right)=\lambda_{2}$, that is, there exists $1 \leq i \leq k, 1 \leq j \leq \ell$ such that $\left(x_{0}, y_{0}\right)=\left(z_{1, i}, z_{2, j}\right)$. Take $x_{n}=\varepsilon_{n} \widetilde{x}_{n}$ and $y_{n}=\varepsilon_{n} \widetilde{y}_{n}$. Then (5.2) holds.

Lemma 5.2 Assume that $\liminf _{|x| \rightarrow \infty} V_{l}(x) \equiv V_{l, \infty}>\lambda_{l}$ for all $l=1,2$. Then there exists a positive number $\varepsilon_{* *}$ such that for every $\varepsilon<\varepsilon_{* *}$, we have $\widehat{M}\left(\varepsilon, \delta_{\varepsilon}\right) \subset \underset{1 \leq i \leq k, 1 \leq j \leq \ell}{\cup} N_{i, j}(\varepsilon)$.

Proof. By Lemma 5.1, we can find $\varepsilon_{* *}>0$ such that for every $\varepsilon<\varepsilon_{* *}$, we have

$$
\sup _{(u, v) \in \widehat{M}\left(\varepsilon, \delta_{\varepsilon}\right)} \inf _{(x, y) \in \mathbf{B}}|(\Phi(u), \Phi(v))-(x, y)|<\frac{s}{2}
$$

or

$$
\operatorname{dist}((\Phi(u), \Phi(v)), \mathbf{B})<\frac{s}{2} \text { for all }(u, v) \in \widehat{M}\left(\varepsilon, \delta_{\varepsilon}\right),
$$

where $\mathbf{B}=\underset{1 \leq i \leq k, 1 \leq j \leq \ell}{\cup}\left[C_{s / 2}\left(z_{1, i}\right) \times C_{s / 2}\left(z_{2, j}\right)\right]$. This implies that

$$
(\Phi(u), \Phi(v)) \in \mathbf{B} \text { for all }(u, v) \in \widehat{M}\left(\varepsilon, \delta_{\varepsilon}\right)
$$

Thus, $\widehat{M}\left(\varepsilon, \delta_{\varepsilon}\right) \subset \underset{1 \leq i \leq k, 1 \leq j \leq \ell}{\cup} N_{i, j}(\varepsilon)$.

Lemma 5.3 Assume that $\lim _{|x| \rightarrow \infty} V_{l}(x)=\lambda_{l}$ for all $l=1,2$. Then

$$
\varepsilon^{-N} c_{\varepsilon}=\widehat{\alpha}_{1}+\widehat{\alpha}_{2} \text { for all } \varepsilon>0
$$

Proof. To prove Lemma 5.3 , we may define the test functions $u_{R}$ and $v_{R}$ by

$$
\begin{aligned}
& \left(u_{R}(x), v_{R}(x)\right) \\
= & \left(\widehat{\omega}_{1}\left(\frac{x+R e}{\varepsilon}\right) \psi_{R}\left(\frac{x+R e}{\varepsilon}\right), \widehat{\omega}_{2}\left(\frac{x-R e}{\varepsilon}\right) \psi_{R}\left(\frac{x-R e}{\varepsilon}\right)\right),
\end{aligned}
$$


where $R>1, e \in S^{N-1}=\left\{x \in \mathbb{R}^{N}:|x|=1\right\}$, $\widehat{\omega}_{l}$ is the unique positive radial solution of Eq. ( $\left.\widehat{E}_{l}\right)$ for $l=1,2$, and the function $\psi_{R} \in C^{1}\left(\mathbb{R}^{N},[0,1]\right)$ with compact support satisfies

$$
\psi_{R}(x)= \begin{cases}1, & |x|<R-1 \\ 0, & |x|>R\end{cases}
$$

and $\left|\nabla \psi_{R}\right| \leq 2$ in $\mathbb{R}^{N}$. Notice that $\widehat{I}_{l}\left(\widehat{\omega}_{l}\right)=\widehat{\alpha}_{1}$ for $l=1,2$. Obviously,

$$
\int_{\mathbb{R}^{N}} \beta(x)\left|u_{R}^{+}\right|^{q+1}\left|v_{R}^{+}\right|^{q+1} \mathrm{~d} x=0 .
$$

By (5.3), it is easy to find two positive numbers $t_{R}$ and $s_{R}$ such that

$$
t_{R}^{2} \int_{\mathbb{R}^{N}}\left(\varepsilon^{2 \alpha}\left|(-\triangle)^{\frac{\alpha}{2}} u_{R}\right|^{2}+V_{1}(x) u_{R}^{2}\right) \mathrm{d} x=\mu_{1} t_{R}^{2 p+2} \int_{\mathbb{R}^{N}}\left|u_{R}^{+}\right|^{2 p+2} \mathrm{~d} x
$$

and

$$
s_{R}^{2} \int_{\mathbb{R}^{N}}\left(\varepsilon^{2 \alpha}\left|(-\triangle)^{\frac{\alpha}{2}} v_{R}\right|^{2}+V_{2}(x) v_{R}^{2}\right) \mathrm{d} x=\mu_{2} s_{R}^{2 p+2} \int_{\mathbb{R}^{N}}\left|v_{R}^{+}\right|^{2 p+2} \mathrm{~d} x .
$$

It follows from (15.4) - (5.6) that $\left(t_{R} u_{R}, s_{R} v_{R}\right) \in \mathbf{N}_{\varepsilon}$ and

$$
J_{\varepsilon}\left(t_{R} u_{R}, s_{R} v_{R}\right) \geq c_{\varepsilon} .
$$

We want to show $\left(t_{R}, s_{R}\right) \rightarrow(1,1)$ as $R \rightarrow \infty$. Using (5.3), (5.5) and change of variables, it is easy to check that

$$
\int_{\mathbb{R}^{N}}\left(\left|(-\triangle)^{\frac{\alpha}{2}} \widehat{\omega}_{1}\right|^{2}+V_{1}(\varepsilon x-R e) \widehat{\omega}_{1}^{2}\right) \mathrm{d} x=\mu_{1} t_{R}^{2 p} \int_{\mathbb{R}^{N}}\left|\widehat{\omega}_{1}^{+}\right|^{2 p+2} \mathrm{~d} x+o_{R}(1),
$$

where $o_{R}(1) \rightarrow 0$ as $R \rightarrow \infty$. Hence, $t_{R} \rightarrow 1$ as $R \rightarrow \infty$ because $\lim _{|x| \rightarrow \infty} V_{1}(x)=\lambda_{1}$ and

$$
\int_{\mathbb{R}^{N}}\left(\left|(-\triangle)^{\frac{\alpha}{2}} \widehat{\omega}_{1}\right|^{2}+\lambda_{1} \widehat{\omega}_{1}^{2}\right) \mathrm{d} x=\mu_{1} \int_{\mathbb{R}^{N}}\left|\widehat{\omega}_{1}^{+}\right|^{2 p+2} \mathrm{~d} x
$$

Similarly, we may obtain $s_{R} \rightarrow 1$ as $R \rightarrow \infty$. Consequently, due to $\lim _{|x| \rightarrow \infty} V_{l}(x)=\lambda_{l}$ for $l=1,2$, we have

$$
\varepsilon^{-N} J_{\varepsilon}\left(t_{R} u_{R}, s_{R} v_{R}\right) \rightarrow \widehat{\alpha}_{1}+\widehat{\alpha}_{2} \text { as } R \rightarrow \infty .
$$

This implies

$$
\varepsilon^{-N} c_{\varepsilon} \leq \widehat{\alpha}_{1}+\widehat{\alpha}_{2} \text { for all } \varepsilon>0 .
$$

Thus, by Lemma 2.3, we have $\varepsilon^{-N} c_{\varepsilon}=\widehat{\alpha}_{1}+\widehat{\alpha}_{2}$ for all $\varepsilon>0$.

We are now ready to prove Theorem 1.3 . Theorem 1.3 can be obtained directly from Lemma 2.3. Lemma 5.2 and Lemma 5.3.

\section{References}

[1] S. Adachi, K. Tanaka, Four positive solutions for the semilinear elliptic equation: $-\triangle u+u=$ $a(x) u^{p}+f(x)$ in $\mathbb{R}^{N}$, Calc. Var. Partial Differ. Equat. 11 (2000) 63-95.

[2] A. Ambrosetti, Critical points and nonlinear variational problems, Bulletin Soc. Math. France, Mémoire, N. 49, 1992.

[3] H. Berestycki, P.L. Lions, Nonlinear scalar field equations. I. Existence of ground state, Arch. Ration. Mech. Anal. 82 (1983) 313-345. 
[4] L. Bergé, Wave collapse in physics: principles and applications to light and plasma waves, Phys. Rep. 303(5-6) (1998) 259-370.

[5] H. Brézis, E.H. Lieb, A relation between pointwise convergence of functions and convergence of functionals, Proc. Amer Math. Soc. 88 (1983) 486-490.

[6] F.E. Browder, Lusternik-Schnirelman category and nonlinear elliptic eigenvalue problems, Bull. Amer. Math. Soc. 71 (1965) 644-648.

[7] D. Cao, E.S. Noussair, Multiplicity of positive and nodal solutions for nonlinear elliptic problems in $\mathbb{R}^{N}$, Ann. Inst. H. Poincaré Anal. Non Lineairé. 13(5) (1996) 567-588.

[8] G. Che, H. Chen, Existence and asymptotic behavior of positive ground state solutions for coupled nonlinear fractional Kirchhoff-type systems, Comput. Math. Appl. (2018) Doi.org/10.1016/j.camwa.2018.09.020.

[9] W. Choi, On strongly indefinite systems involving the fractional Laplacian, Nonlinear Anal. 120 (2015) 127-153.

[10] R. Cipolatti, W. Zumpichiatti, On the existence regularity of ground state for a nonlinear system of coupled Schrödinger equations in $\mathbb{R}^{N}$, Comput. Appl. Math. 18 (1999) 15-29.

[11] G. Chen, Y. Zheng, Concentration phenomenon for fractional nonlinear Schrödinger equations, Commun. Pure Appl. Anal. 13 (2014) 2359-2376.

[12] S. Dipierro, A. Pinamonti, A geometric inequality and a symmetric result for elliptic systems involving the fractional Laplacian, J. Differential Equations. 255 (2013) 85-119.

[13] E. Di Nezza, G. Palatucci, E. Valdinoci, Hitchhiker's guide to the fractional Sobolev spaces, Bull. Sci. Math. 136 (2012) 521-573.

[14] J. Daila, M. del Pin, J. Wei, Concentrating standing waves for the fractional nonlinear Schrödinger equation, J. Differential Equations. 256 (2014) 858-892.

[15] I. Ekeland, On the variational principle, J. Math. Anal. Appl. 17 (1974) 324-353.

[16] B. Esry, C. Greene, J. Burke Jr, J. Bohn, Hartree-Fock theory for double condensates, Phys. Rev. Lett, 78 (1997) 3594-3597.

[17] M. M. Fall, E. Valdinci, Uniqueness and nondegeneracy of positive solutions of $(-\triangle)^{s} u+u=u^{p}$ in $\mathbb{R}^{N}$ when $s$ is close to 1 , Comm. Math. Phys. 329 (2014) 383-404.

[18] P. Felmer, A. Quass, J. Tan, Positive solutions of the nonlinear Schrödinger equation with the fractional Laplacian, Proc. Roy. Soc. Edinburgh Sect. A. 142 (2012) 1237-1262.

[19] G. Fibich, G. Papanicolaou, Self-focusing in the perturbed and unperturbed nonlinear Schrödinger equation in critical dimension, SIAM J. Appl. Math. 60 (2000) 183-240.

[20] R.L. Frank, E. Lenzmann, L. Silvestre, Uniqueness of radial solutions for the fractional Laplacian, Commun. Pure Appl. Math. 69(9) (2016) 1671-1726.

[21] D.G. de Figueiredo, O. Lopes, Solitary waves for some nonlinear Schrödinger systems, Ann. Inst. H. Poincaré Anal. Non Linéaire. 25 (2008) 149-161.

[22] M. M. Fall, F. Mahmoudi, E. Valdinoci, Ground states and concentration phenomena for the fractional Schrödinger equation, Nonlinearity 28 (2015) 1937-1961. 
[23] Q. Guo, X. He, Semiclassical states for weakly coupled fractional Schrödinger system, J. Differential Equations. 263 (2017) 1986-2023.

[24] Q. Guo, X. He, Least energy solutions for a weakly coupled fractional Schrödinger system, Nonlinear Anal. 132 (2016) 141-159.

[25] D. Hall, R. Matthews, J. Ensher, C. Wieman, E. Cornell, Dynamics of component separation in a binary mixture of Bose-Einstein condensates, Phys. Rev. Lett. 81(8) (1998) 515-518.

[26] N. Ikoma, Uniqueness of positive solutions for a nonlinear elliptic system, Nonlinear Differ. Equ. Appl. 16 (2009) 555-567.

[27] M.K. Kwong, Uniqueness of positive solutions of $\Delta u-u+u^{p}=0$ in $\mathbb{R}^{N}$, Arch. Rational Mech. Anal. 105 (1989) 243-266.

[28] M. Lakshmanan, T. Kanna, R. Radhakrishnan, Sharp-changing collisions of coupled bright solitions in birefringent optical fibers, Rep. Math. Phys. 46 (2000) 143-156.

[29] N. Laskin, Fractional quantum mechanics and Lévy path integral, Phys. Lett. A. 268 (2000) 298-305.

[30] N. Laskin, Fractional Schrödinger equation, Phys. Rev. E. 66 (2002) 056108, 7pp.

[31] T.C. Lin, J. Wei, Spikes in two coupled nonlinear Schrödinger equations, Ann. I. H. Poincaré Anal. Non Linéaire. 22 (2005) 403-439.

[32] T.C. Lin, J. Wei, Spikes in two-component systems of nonlinear Schrödinger equations with trapping potentials, J. Differential Equations. 229 (2006) 538-569.

[33] T.C. Lin, T.F. Wu, Existence and multiplicity of positive solutions for two coupled nonlinear Schrödinger equations, Discrete Contin. Dyn. Syst. 33 (2013) 2911-2938.

[34] P.L. Lions, The concentration-compactness principle in the calculus of variations. The locally compact case part 1, Ann. Inst. H. Poincaré Anal. Non Linéaire. 1(1) (1984) 109-145.

[35] P.L. Lions, The concentration-compactness principle in the calculus of variations. The locally compact case part 2, Ann. Inst. H. Poincaré Anal. Non Linéaire. 1(2) (1984) 223-283.

[36] C.H. Liu, H.Y. Wang, T.F. Wu, Multiplicity of 2-nodal solutions for semilinear elliptic problems in $\mathbb{R}^{N}$, J. Math. Anal. Appl. 348 (2008) 169-179.

[37] D. Lü, S. Peng, On the positive vector solutions for nonlinear fractional Laplacian system with linear coupling, Discrete Contin. Dyn. Syst. 37(6) (2017) 3327-3352.

[38] L.A. Maia, E. Montefusco, B. Pellacci, Positive solutions for a weakly coupled Schrödinger system, J. Differential Equations. 229 (2006) 743-767.

[39] S. Peng, H. Pi, Spike vector solutions for some coupled nonlinear Schrödinger equations, Discrete Contin. Dyn. Syst. 36 (4) (2016) 2205-2227.

[40] S. Secchi, Ground state solutions for nonlinear fractional Schrödinger equations in $\mathbb{R}^{N}$, J. Math. Phys. 54 (2013) 031501.

[41] L. Silvestre, Regularity of the obstacle problem for a fractional power of the Laplace operator, Dissertation, University of Texas at Austin, (2005). 
[42] E. Timmermans, Phase seperation of Bose-Einstein condensates, Phys. Rev. Lett. 81 (1998) $5718-5721$.

[43] M. Willem, Minimax Theorems, Birkhäuser, Boston, 1996. 